\title{
Gender differences in circulating levels of neutrophil extracellular traps in serum of multiple sclerosis patients
}

Tillack, K ; Naegele, M ; Haueis, C ; Schippling, S ; Wandinger, K P ; Martin, R ; Sospedra, M

\begin{abstract}
Neutrophil extracellular traps (NETs) trap and kill pathogens very efficiently but also activate dendritic cells and prime T cells. Previously, we demonstrated that neutrophils are primed and circulating NETs are elevated in relapsing remitting multiple sclerosis (RRMS), a T cell-mediated autoimmune disease. Here, we demonstrate gender specific differences in circulating NETs but not in neutrophil priming in RRMS patients. Although the results from our systematic and in depth characterization of these patients argue against a major role of circulating NETs in this disease, they suggest that NETs may underlie gender-specific differences in MS pathogenesis.
\end{abstract}

DOI: https://doi.org/10.1016/j.jneuroim.2013.05.004

Posted at the Zurich Open Repository and Archive, University of Zurich

ZORA URL: https://doi.org/10.5167/uzh-86853

Journal Article

Accepted Version

Originally published at:

Tillack, K; Naegele, M; Haueis, C; Schippling, S; Wandinger, K P; Martin, R; Sospedra, M (2013). Gender differences in circulating levels of neutrophil extracellular traps in serum of multiple sclerosis patients. Journal of Neuroimmunology, 261(1-2):108-119.

DOI: https://doi.org/10.1016/j.jneuroim.2013.05.004 


\section{Gender Differences in Circulating Levels of Neutrophil Extracellular Traps in Serum of Multiple Sclerosis Patients}

\section{Kati Tillack ${ }^{1}$, Matthias Naegele ${ }^{1}$, Cathleen Haueis ${ }^{1}$, Sven Schippling ${ }^{1,2}$, Klaus-Peter Wandinger ${ }^{1,3}$, Roland Martin ${ }^{1,2}$, Mireia Sospedra ${ }^{1,2, *}$.}

${ }^{1}$ Institute for Neuroimmunology and Clinical MS Research (inims), Center for Molecular Neurobiology (ZMNH), University Medical Center Hamburg-Eppendorf, Falkenried 94, 20251 Hamburg, Germany.

${ }^{2}$ Neuroimmunology and MS Research, Department of Neurology, University Zurich, Frauenklinikstrasse 26, 8091 Zurich, Switzerland.

${ }^{3}$ Institute of Clinical Chemistry, University Medical-Center Schleswig-Holstein Campus Lübeck, Ratzeburger Allee 160, 23538 Lübeck, Germany.

${ }^{*}$ Corresponding author:

Mireia Sospedra

Neuroimmunology and MS Research

Department of Neurology

University Zurich

Frauenklinikstrasse 26

8091 Zürich

Switzerland

Phone: + 41442553905

Fax: +41442558864

e-mail: Mireia.SospedraRamos@usz.ch

Running title: Circulating NETs in MS patients 


\section{Abstract (100 words)}

Neutrophil extracellular traps (NETs) trap and kill pathogens very efficiently but also activate dendritic cells and prime $\mathrm{T}$ cells. Previously, we demonstrated that neutrophils are primed and circulating NETs are elevated in relapsing remitting multiple sclerosis (RRMS), a $\mathrm{T}$ cell-mediated autoimmune disease. Here, we demonstrate gender specific differences in circulating NETs but not in neutrophil priming in RRMS patients. Although the results from our systematic and in depth characterization of these patients argue against a major role of circulating NETs in this disease, they suggest that NETs may underlie gender-specific differences in MS pathogenesis.

Key words: Neutrophil, neutrophil extracellular traps (NETs), multiple sclerosis, gender 


\section{1-Introduction}

The main function of the innate immune system during infection is to eliminate the pathogen, and neutrophils are key players in innate immune responses. Women of reproductive age are more resistant to sepsis and subsequent morbidity and mortality than men (Schroder et al., 1998), and the incidence of sepsis in postmenopausal women increases to levels almost equal to those seen in age-matched men (Martin et al., 2003). Women also have a higher systemic neutrophil count compared with men (Bain and England, 1975a), and neutrophil counts correlate with estradiol levels during menstruation (Bain and England, 1975b) and pregnancy (Efrati et al., 1964) suggesting that sex hormones influence neutrophilia and overall resistance to sepsis most likely by delaying apoptosis in neutrophils (Molloy et al., 2003). In order to eliminate pathogens during infections, neutrophils are armed with a variety of weapons including engulfment and intracellular degradation of microbes (Hampton et al., 1998; Segal, 2005), release of oxygen species and granule proteins (Lehrer and Ganz, 1999) and release of extracellular chromatin fibers bound to granular, nuclear and cytoplasmic proteins called neutrophil extracellular traps (NETs) (Brinkmann et al., 2004). NETs not only trap and kill pathogens very efficiently but also minimize collateral tissue damage by containing proteases to the DNA fibers and act as physical barriers preventing microbial spread. Despite the well documented importance of NETs as an effective antimicrobial first line defense mechanism, there is increasing evidence that NETs occur in various clinical settings in the absence of microbial infections and that they are probably also associated with pathophysiological conditions (Amulic et al., 2012; Logters et al., 2009). NETosis, the process of neutrophil cell death that leads to expulsion of NETs, can be triggered by different stimuli including pro-inflammatory cytokines such as IL-8 and TNF $\alpha$, phorbol 12myristate 13-acetate (PMA) (Brinkmann et al., 2004; Fuchs et al., 2007), activated platelets (Clark et al., 2007) and endothelial cells (Gupta et al., 2010), and placental microparticles (Gupta et al., 2005). In addition to their bactericidal potential, NETs can also activate plasmacytoid- (pDCs) (GarciaRomo et al., 2011; Lande et al., 2011) and myeloid (mDC) (Sangaletti et al., 2012) dendritic cells and in consequence modulate inflammatory responses. 
The activation of DCs by NETs appears to play an important role in the pathogenesis of some autoimmune diseases such as psoriasis (Lande et al., 2007), systemic lupus erythematosus (SLE) (Barrat et al., 2005; Garcia-Romo et al., 2011; Lande et al., 2011; Means et al., 2005) small vessel vasculitis (Sangaletti et al., 2012) and type I diabetes (Diana et al., 2013). NETsactivated pDCs produce large amounts of interferon that can lead to the maturation of myeloid DCs (mDCs) and exert an effect on T cell function. In SLE, pathogenic auto-antibodies have been associated with abnormal clearing of NETs and NETosis that results in abnormally high production and/or low degradation of NETs, which then leads to tissue damage and facilitated generation of large quantities of auto-antibodies creating a vicious cycle (Knight and Kaplan, 2012). In small vessel vasculitis, NETs can favor neutropil proteins uploading into $\mathrm{mDCs}$ and the induction of anti-neutrophil cytoplasmic antibodies (ANCAs) (Sangaletti et al., 2012). Recently, we demonstrated that NETs are also able to directly prime $T$ cells by reducing their activation threshold, which represents a novel link between innate and adaptive immune responses (Tillack et al., 2012).

Multiple sclerosis (MS) is a chronic inflammatory, demyelinating disease of the central nervous system with aspects of secondary neurodegeneration (Sospedra and Martin, 2005). There are two major forms of MS, relapsingremitting (RR)-MS, which affects around $85 \%-90 \%$ of patients, and primary progressive (PP)-MS in around 10\%-15\%. Most RR-MS patients later develop secondary progressive (SP)-MS. It is not clear at present, which factors are responsible for the different courses. RRMS affects women about twice as often as men, while no gender differences are observed in PPMS. The activation of $\mathrm{CD}^{+}$autoreactive $\mathrm{T}$ cells and their differentiation into a T-helper type 1 (Th1) phenotype is a crucial event both in the initial phase and in the long-term evolution of RRMS. Damage of the central nervous system is however, most likely mediated by antibodies, complement, $\mathrm{CD} 8^{+} \mathrm{T}$ cells, and factors produced by innate immune cells. Deregulation in other immune networks involving T-helper 2 (Th2) cells, regulatory CD4 ${ }^{+} \mathrm{T}$ cells or NK cells are probably also involved. Compared to $\mathrm{T}$ cell- and antibody responses or to other innate immune cells such as DCs and monocytes, the role of neutrophils 
in MS has not been examined extensively. In a previous study (Naegele et al., 2012), we demonstrated that neutrophils in RRMS patients are more numerous and exhibit a primed state. Furthermore we found higher levels of NETs in serum from these patients. Gender specific differences were not addressed in this study. The chronic inflammatory environment in RRMS (Lund et al., 2004) probably underlies this inappropriate neutrophil priming, which may result in enhanced neutrophil activation during infection. The higher levels of NETs in serum might also be linked to the chronic inflammatory environment in MS, but may have been caused by other triggers such as infections, that have been associated with relapses in these patients (Granieri et al., 2001) or higher frequency of activated platelets (Sheremata et al., 2008) or endothelial cells (Minagar et al., 2001) both elevated in MS. Due to the central role of $T$ cells in RRMS and the ability of NETs to modulate $T$ cell immunity, an abnormally gender-specific high level of NETs in this disease as consequence of neutrophil priming may play an important role in certain aspects of the pathogenesis and also explain some gender-specific differences.

Here, we have analyzed in detail a large cohort of MS patients including those with subforms other than RRMS, and we have found that only a subset of RRMS patients shows elevated circulating NETs. We also examined whether the higher levels of NETs as well as the primed state of neutrophils in these patients show sex differences, and our results indeed indicate that gender specific differences exist with respect to levels of circulating NETs but not in neutrophil priming. Further, we have performed a systematic and in depth characterization of these patients aimed to identify NETs triggers as well as their putative role in MS pathogenesis. The results argue against a major role of circulating NETs in MS but suggest that NETs may underlie gender-specific differences in MS pathogenesis. 


\section{2-Material and Methods}

\subsection{Patients}

Patients were recruited from the inims outpatient clinic and day hospital at the University Medical Center Hamburg-Eppendorf. MS diagnosis was based on the revised McDonald criteria (Polman et al., 2005). Patients, who had not received steroids for at least 4 weeks prior to enrolment or any immunomodulatory or immunosuppressive agent during the last 3 months, were considered untreated and included in the study. Patients were classified into the following MS subgroups: patients with a first demyelinating event suggestive of MS, termed clinically isolated syndrome (CIS, $n=126)$, RRMS $(n=168)$ and PPMS $(n=21)$. The CIS and RRMS group included patients that were either neurologically stable for at least 30 days before sampling (remission state, CIS n=76 and RRMS n=99) or exhibited an acute episode of neurological worsening lasting for more than $24 \mathrm{~h}$ at the time of sampling and not having received steroids (relapse state, $\operatorname{CIS} n=50$ and RRMS $n=69$ ). Patients did not show clinical signs or symptoms of acute infection. Controls included patients with inflammatory neurological disease other than MS (OIND, $n=5$ ), patients with other non-inflammatory neurological diseases (OND, $n=12$ ) and a cohort of healthy volunteers not suffering from any known infectious or inflammatory disorder $(\mathrm{HC}, \mathrm{n}=40)$. Table 1 summarizes the demographic characteristics of patients and controls. Supplementary Table 1 summarizes the diagnosis of patients with OIND and OND. This study was approved by the local ethics committee (Ethik-Kommission der Ärztekammer Hamburg), and written informed consent was obtained from all patients and controls before blood was drawn.

\subsection{Quantification of circulating NETs and dsDNA in serum samples}

Detection of myeloperoxidase (MPO) associated with DNA was used to quantify circulating NETs. MPO-DNA complexes in serum samples were quantified as previously described (Kessenbrock et al., 2009). Briefly, $5 \mu \mathrm{g} / \mathrm{ml}$ of mouse anti-human myeloperoxidase (MPO)-specific capture antibody (AbD SeroTec, Duesseldorf, Germany) was coated to 96-well plates. After blocking with $1 \%$ BSA, serum samples were added together with a peroxidase-labeled 
anti-DNA monoclonal antibody (component 2 of the Cell Death ELISA kit, Roche). After $2 \mathrm{~h}$ of incubation, the peroxidase substrate was added according to manufacturer's instructions. Absorbance was measured at 405 $n m$ using a $\mu$ Quant microplate reader (Bio-Tek, Winooski, VT, USA). Total dsDNA in serum was quantified using Picogreen dsDNA kit (invitrogen, GIBCO, Paisley, UK) as previously described (Fuchs et al., 2007).

\subsection{Neutrophil purification and in vitro stimulation}

Neutrophils from RRMS patients and $\mathrm{HC}$ were isolated from freshly drawn peripheral blood using Dextran-Ficoll, as described previously (Weiss et al., 1985). Briefly, erythrocytes were sedimented for 30 minutes in Hank's balanced salt solution (HBSS) (Invitrogen GIBCO, Paisley, UK) containing 3\% pure Dextran 200 (Serva, Heidelberg, Germany). The top phase was carefully layered onto $3 \mathrm{ml}$ of LSM 1077 Ficoll solution (PAA Laboratories $\mathrm{GmbH}$, Pasching, Austria) and centrifuged for 30 minutes at $2000 \mathrm{rpm}$ without brakes. After centrifugation, the supernatant was discarded and the pellet washed. Remaining erythrocytes were lysed by adding $5 \mathrm{ml}$ ice-cold $\mathrm{H}_{2} \mathrm{O}$ for $20 \mathrm{sec}$. Neutrophil purity and viability was $\geq 97 \%$ and $\geq 95 \%$ respectively as assessed by expression of the neutrophil-specific marker CD16b and trypan blue exclusion as already described (Naegele et al., 2012).

Purified neutrophils were resuspended in HBSS (Invitrogen) medium supplemented with $10 \mathrm{mM}$ Hepes (Invitrogen). $5 \times 10^{5}-10^{6}$ neutrophils $/ \mathrm{ml}$ were seeded into tissue culture plates on glass coverslips (Menzel, $\mathrm{GmbH}$, Braunschweig, Germany) pretreated with $0.001 \%$ poly-L-lysine (SigmaAldrich, Steinheim, Germany). Neutrophils were stimulated with $25 \mathrm{nM}$ PMA (Sigma-Aldrich) for $3 \mathrm{~h}$.

\subsection{Isolation and quantification of NETs}

NETs released by activated neutrophils were digested with $10 \mathrm{U} / \mathrm{ml}$ micrococcal nuclease (MNase, Worthington Biochemical Corp. Lakewood, $\mathrm{NJ}$ ) as previously described (Urban et al., 2009). NETs (MPO-DNA complexes) in supernatants were quantified using a capture ELISA as previously described (Kessenbrock et al., 2009). Absorbance was measured at $405 \mathrm{~nm}$ using a $\mu$ Quant microplate reader (Bio-Tek, Winooski, VT, USA). 


\subsection{Protein- and cytokine determinations}

C-reactive protein (CRP) and IL-6 levels in serum samples were measured at the central laboratory, University Medical Center Hamburg-Eppendorf. IL-8, platelet factor 4 (PF4), beta-thromboglobulin (beta-TG) and sVE-cadherin levels in serum samples were measured by ELISA using the following kits: Human IL-8 CytoSet $^{\mathrm{TM}}$ (Biosource, Invitrogen), sCD144 (sVE-Cadherin) ELISA kit (PromoKine, PromoCell, GmbH, Germany), Asserumchrom® ${ }^{\circledR}$ TG (Diagnostica Stago, Parsippany, NJ) and RayBio® Human PF-4 ELISA kit (RayBiotech, Norcross, GA). Reactions were performed according to the manufacturer's instructions.

\subsection{Blood cell counts}

Peripheral blood was collected in EDTA-tubes and cell counts determined using an AcT Diff Coulter Counter (Beckman Coulter, Inc. Fullerton, CA) standardized with Coulter 4C-ES Cell Control (Beckman Coulter).

\subsection{Intracellular cytokine staining}

For intracellular cytokine staining, PBMCs were stimulated with PMA (50 $\mathrm{ng} / \mathrm{ml}$, Sigma) and ionomycin ( $1 \mu \mathrm{g} / \mathrm{ml}$, Sigma) in the presence of Brefeldin A $\left(10 \mu \mathrm{g} / \mathrm{ml}\right.$, eBioscience) for $5 \mathrm{~h}$. Next, cells were stained with LIVE/DEAD ${ }^{\circledR}$ Fixable Dead Cell Stain Kit (AmCyan, Molecular Probes, Invitrogen), fixed and permeabilized with the corresponding buffers (eBioscience), and stained for CD3 (PE, DakoCytomation, Denmark), CD8 (PB, DakoCytomation, Denmark), IFN $\gamma$ (FITC, BDPharmingen), IL-4 (PE-Cy7, eBioscience) and IL-17A (Alexa Fluor ${ }^{\circledR}-647$, eBioscience) at room temperature. Sample acquisition was done with a LSR-II (BD) flow cytometer and data analyzed with FACS Diva (BD) and the FlowJo (Tree Star, Inc.) software packages.

\subsection{Characterization of peripheral blood monocytes and dendritic cells}

Human PBMCs were isolated from peripheral venous blood by density gradient and stained with anti-CD14 (Pacific Blue, Becton Dickinson, BD, Franklin Lakes, NJ), anti-CD16 (APC-Cy7, Biozol, Germany), anti-CD19 (Pacific Blue, BD) and anti-CD40 PE (PE, Miltenyi, Biotec, GmbH, Germany) for the characterization of monocytes and with anti-CD14, anti-CD19, anti- 
CD40, anti-CD86 (FITC, Dako), anti-CD141 (APC, Miltenyi), anti-CD303 (APC, Miltenyi) and anti-CD1c (APC, Miltenyi) for the characterization of dendritic cells. Corresponding isotype controls were also measured. Sample acquisition was done with a LSR-II (BD) flow cytometer and data analyzed with FACS Diva (BD) and FlowJo (Tree Star, Inc.) software packages.

\subsection{Autoantibodies determination}

Anti-nuclear antibodies (ANAs), anti-neutrophil cytoplasmic antibodies (ANCAs) and anti-ds DNA titer in serum samples were determined using commercial assays (Euroimmun, Luebeck, Germany).

\subsection{Statistical analysis}

Statistical analyses were performed with Prism 5.02 (GraphPad Software Inc., San Diego, CA). Parametric tests were applied for two-group comparisons using unpaired t-tests with two-tailed $p$-values. Comparisons of three groups and more were assessed by one-way ANOVA with Bonferroni's correction for multiple comparisons. P-values $<0.05$ were considered statistically significant. 


\section{3-Results}

\subsection{Gender differences in circulating levels of NETs in serum of RRMS patients}

In order to explore whether higher levels of circulating NETs are a unique feature of RRMS patients, we quantified MPO-DNA complexes in serum of other MS and non-MS patients including CIS $(n=126)$, PPMS $(n=21)$, OIND $(n=5)$ and OND $(n=10)$ patients. Significant differences in the level of MPODNA complexes were observed in RRMS and OIND patients but not in CIS, PPMS or OND patients compared with HC (Fig. 1A). The amount of MPODNA complexes in serum of RRMS patients showed strong inter-individual variability, with optic density (OD) at $405 \mathrm{~nm}$ ranging from 0.107 to 2.288 . In order to facilitate analysis, we have arbitrarily defined a threshold of $O D>0.3$ to identify patients with high content of MPO-DNA complexes in serum. 17.7 $\%$ of RRMS and $60 \%$ of OIND patients showed OD $>0.3$, i.e. high levels of MPO-DNA complexes in serum. The percentage of HC (7.5\%), CIS (8.8\%), PPMS (4.7\%) or OND (10\%) patients belonging to this group was lower (Fig. $1 A)$.

We also analyzed whether MPO-DNA complexes were present in CSF samples of CIS ( $n=20)$, RRMS ( $n=21)$, PPMS (6), OIND (5) and OND (10) patients. No MPO-DNA complexes were detectable in CSF (Fig. 1B).

MPO-DNA complexes did not correlate with age (Fig. 1C) but unexpectedly were significantly higher in males compared with females (Fig. 1D). When the different groups of patients and $\mathrm{HC}$ were analyzed independently, sex-specific differences were statistically significant only in RRMS patients. $12.1 \%$ of female RRMS patients showed OD >0.3, i.e. high levels of MPO-DNA complexes in serum, compared to $26.2 \%$ of male RRMS patients (Fig. 1D). Interestingly, our previous observation that neutrophils in RRMS patients are more numerous and exhibit a primed state does not seem to underlie the gender-specific differences in circulating levels of MPO-DNA complexes, since neutrophil counts, neutrophil expression of fMLP receptor (FPR1), IL-8 receptor (CXCR1), TLR2, CD43 and CD63, all markers of neutrophil priming 
and significantly higher in RRMS patients (Naegele et al., 2012), did not show gender-specific differences (Supplementary Fig. 1).

\subsection{Gender differences in NETs formation and degradation}

In order to explore whether NETosis can be more easily induced in male RRMS neutrophils, which could explain the gender-specific differences in MPO-DNA complexes in serum, we compared the ability of purified neutrophils from female and male RRMS patients and $\mathrm{HC}$ to release NETs in vitro. Purified neutrophils from female $(n=20)$ and male $(n=10)$ RRMS patients and female $(n=33)$ and male $(n=22)$ HC were stimulated in vitro with PMA and NETs were isolated and quantified using an MPO-DNA capture ELISA. Results are summarized in Figure 2A. No significant differences in NET formation were observed between neutrophils from female and male RRMS patients and $\mathrm{HC}$, although females from both groups showed a tendency to release more MPO-DNA complexes than males.

Next, in order to examine whether gender differences in DNAse activity could be involved in the high level of MPO-DNA complexes in male RRMS patients, we have compared cell-free dsDNA in serum of pre-selected groups of female and male RRMS and CIS patients, each group containing a similar number of individuals with high $(O D>0.3$, RRMS female $n=9$ and male $n=9$, CIS female $\mathrm{n}=7$, males=7) and low (OD $<0.2$, RRMS female $\mathrm{n}=9$ and male $\mathrm{n}=9$, CIS female $n=6$, males=8) levels of MPO-DNA complexes (Fig. 2B). No correlation between NETs and dsDNA was observed in these groups of patients, but interestingly, male RRMS- but not CIS patients showed a significantly higher level of cell free dsDNA in serum compared with females (Fig. 2B). When the level of dsDNA in female and male RRMS and CIS patients containing high or low levels of MPO-DNA complexes was compared (Fig. 2C), a significantly higher level of dsDNA was found in serum of male RRMS with high MPODNA complexes compared with females with low complexes, while no differences were found in CIS patients.

\subsection{Circulating NETs in serum and disease activity}

In order to characterize circulating levels of NETs in serum of RRMS patients, we measured MPO-DNA complexes in different serum samples obtained from 
8 individual patients ( 4 females and 4 males) at different time points (Fig. 3A). We found that high levels of NETs $(O D>0.3)$ were not stable but fluctuated over time. In some patients the analyzed serum samples never reached an $\mathrm{OD}>0.3$, indicating low levels of circulating NETs. Interestingly, patient 2 showed a very high level of circulating NETs over a period of 30 months.

With the purpose to examine whether these fluctuations were related with disease activity, we compared serum samples of females and males $\mathrm{CIS}$ and RRMS patients in remission and relapse (CIS females $n=40$ remission and 38 relapse, CIS males $n=29$ remission and 19 relapse; RRMS females $n=63$ remission and 43 relapse, RRMS males $n=36$ remission and 26 relapse). No significant differences in the amounts of MPO-DNA complexes were observed between patients in remission and relapse (Fig. 3B). We also analyzed 22 RRMS patients, from whom paired serum samples corresponding to relapse and remission were available (Fig. $3 \mathrm{C}$ ). In most patients higher or similar amounts of MPO-DNA complexes were detected in relapse while only in a few patients the amount was higher during remission.

\subsection{Putative triggers of NET formation in RRMS patients}

To address a putative link between NETs release and inflammation, we compared the level of serum IL-8 as an indicator of inflammation in preselected groups of female and male RRMS patients with either high (OD > 0.3 , females $n=9$, males $n=9$ ) or low ( $O D<0.2$, females $n=9$, males $n=9$ ) levels of MPO-DNA complexes. As shown in Figure 4, no significant differences were observed between the different groups of patients. We also explored a putative role of infections in NETs release, by measuring Creactive protein (CRP) and IL-6. CRP is an acute-phase protein that rises in response to inflammation, and IL-6 is also an important mediator of acutephase responses. No significant differences in CRP or IL-6 levels between the different groups of patients were found (Fig. 4). Finally, we also examined a putative role of platelet and endothelial activation in NETosis by measuring the levels of platelet activation factor (PF4) and soluble beta-thromboglobulin (beta-TG) (Fig. 4) as markers of platelet activation (Kaplan and Owen, 1981; Stuckey et al., 1992) and soluble sVE cadherin (CD144, Fig. 4) as marker of endothelial activation and damage (Vestweber, 2008). No significant 
differences in PAF, beta-TG or sVE cadherin levels between the different groups of patients were observed.

\subsection{Circulating NETs and peripheral blood lymphocytes}

Next, in order to explore a putative role of NETs in gender-specific differences in MS pathogenesis, we examined whether gender as well as the level of MPO-DNA complexes in serum have an effect on the circulating number of lymphocytes. First we compared the absolute lymphocyte counts in whole blood of females and males RRMS and HC (RRMS females $n=35$, males $n=20 ; \mathrm{HC}$ females $n=25$, males $n=15$ ), and no differences were found (Fig. $5 A)$. Then we measured absolute lymphocyte counts in female and male RRMS patients with high (OD > 3; females $n=12$, males $n=9$ ) and low (OD < 0.2; females $n=13$, males $n=10$ ) MPO-DNA complexes in serum. Again no significant differences were found between the different groups (Fig. 5B). Circulating lymphocytes were further characterized by flow cytometry for a putative effect of gender and circulating MPO-DNA complexes on their functional phenotype. The percentages of $\mathrm{CD} 8^{+}$IFN $\gamma$-producing cells as well as of $\mathrm{CD}^{+}$Th1 (IFN $\gamma$-producing), Th2 (IL-4-producing) or Th17 (IL-17producing) cells were measured by intracellular cytokine staining in PBMCs of female and male RRMS patients with high (OD > 3; females $n=8$, males $n=8$ ) and low (OD < 0.2; females $n=8$, males $n=8$ ) MPO-DNA complexes in serum. No differences in the percentage of any of these $T$ cell populations were found between the different groups of patients (Fig. 5C and D).

\subsection{Circulating NETs and peripheral blood dendritic cells}

Since it has been demonstrated that NETs can activate DCs, we also examined whether gender and the level of MPO-DNA complexes in serum have an effect on circulating DCs. We measured by flow cytometry the percentages of plasmacytoid, myeloid type 1 and myeloid type 2 DCs as well as their expression of CD40 and CD86 in female and male RRMS patients with high $(O D>0.3$; females $n=15$, males $n=13$ ) and low $(O D<0.2$; females $n=14$, males $n=13$ ) MPO-DNA complexes in serum (Fig. 6). We did not find significant differences in the percentage of any of these DC populations nor in their activation state between the different groups of patients. 


\subsection{Circulating NETs and peripheral blood monocytes}

Subsequently, we also examined whether gender and level of MPO-DNA complexes in serum have an effect on circulating monocytes. First, we compared the absolute monocyte counts in whole blood of females and males RRMS and HC (RRMS females $n=35$, males $n=20 ; H C$ females $n=25$, males $n=15$ ), and no differences were found (Fig. 7A). Then we measured absolute monocyte counts in female and male RRMS patients with high (OD > 3; females $n=12$, males $n=9$ ) and low ( $O D<0.2$; females $n=13$, males $n=10$ ) MPO-DNA complexes in serum. Unexpectedly, significantly higher monocyte counts were found in RRMS patients with high MPO-DNA complexes in serum, and interestingly the strong difference was observed between males with high and females with low MPO-DNA complexes in serum (Fig. 7B). In order to better understand the putative effect of gender and NETs on monocyte expansion, we also measured by flow cytometry in PBMCs the percentage of classical, intermediate and non-classical monocytes as well their expression of CD40 in female and male RRMS patients with high (OD > 0.3 ; females $n=10$, males $n=7$ ) and low ( $O D<0.2$; females $n=9$, males $n=7$ ) MPO-DNA complexes in serum. We did not find significant differences in the percentage of any of these monocyte populations and neither regarding their activation state (Fig. 7C).

\subsection{Autoantibodies in RRMS patients with NETs in serum}

Finally, since pathogenic antinuclear (ANA), ANCA and anti-dsDNA autoantibodies have been associated with abnormal NETs clearing and NETosis in SLE (Knight and Kaplan, 2012), anti-dsDNA auto-antibodies are more prevalent among male SLE patients, and further since ANCAs have been found elevated in patients with the optico-spinal form of MS in Japan (Fukazawa et al., 1996), we measured ANA, cANCA, pANCA and anti-dsDNA antibody levels in serum samples of female and male RRMS patients with high ( $O D>3$; females $n=6$, males $n=5$ ) and low (OD < 0.2; females $n=6$, males $n=4)$ MPO-DNA complexes. No anti-dsDNA nor cANCA autoantibodies were detected in any sample and for ANA and CANCA, although a tendency to higher levels in males was observed, differences did not reach significance (Table 2). 


\section{4-Discussion}

NETs are extracellular chromatin fibers bound to granular, nuclear and cytoplasmic proteins that despite their importance as effective antimicrobial first line defense mechanism, also occur in various clinical settings in the absence of microbial infections and might be associated with pathophysiological conditions (Logters et al., 2009). In a previous study (Naegele et al., 2012), we demonstrated that neutrophils in RRMS patients are more numerous and exhibit a primed state. Among the features of neutrophils in these patients, we found significantly higher levels of NETs in the serum and speculated that these may be involved in MS pathogenesis. To examine this issue in depth, we analyzed here a large cohort of MS patients including patients suffering from subforms of MS other than RRMS, and found that only a small subset of RRMS patients have elevated circulating NETs. In view of the fact that women of reproductive age have a higher systemic neutrophil count compared with men and that RRMS affects women about twice as often as men we then examined whether the level of NETs in serum as well as neutrophil counts and primed phenotype in RRMS patients were higher in females. Unexpectedly, our results demonstrate that the subset of RRMS patients with high NETs in serum was significantly enriched in male patients while no gender specific differences were observed in neutrophil counts and primed state. Although higher circulating NET levels do not seem to be a general feature of RRMS patients, they might still be relevant in the above subset of RRMS patients and underlie gender-specific differences in MS pathogenesis. MS is a highly heterogeneous disease most likely involving very diverse pathomechanisms. Neutrophils are normally not present in the CNS of MS patients, and consistent with this notion we did not detect NETs in CSF samples from these patients. Considering the potential dangers associated with the indiscriminate histotoxic potential of neutrophils there may be active mechanisms preventing their entry into the vulnerable CNS compartment. However, these cells are likely to play a role in opening the blood brain barrier, particularly via NETosis, and this potential mechanism deserves further investigation. 
The higher circulating NETs in a subset of RRMS patients and particularly in males could reflect an alteration in NET formation and/or in NET degradation. Neutrophils in RRMS patients are primed and can easily be activated (Naegele et al., 2012), which could explain higher NETosis in RRMS patients. However, our observation that granulocyte counts as well as the neutrophil primed state did not show gender-specific differences argues against this hypothesis. To explore further this possibility, we also analyzed NETosis in vitro using purified neutrophils from female and male RRMS patients and HC. We did not find differences in NETosis between purified neutrophils from RRMS and $\mathrm{HC}$ and neither gender-specific significant differences. In contrast, male RRMS patients showed significantly higher cell-free dsDNA in serum compared with female patients suggesting a putative abnormal DNAse activity that might underlie the higher circulating NETs levels in male RRMS patients. Interestingly, in SLE, in which women are affected about 10 times more frequently than men, it is interesting to note that male gender has been associated with more severe disease and with higher prevalence of pathogenic anti-dsDNA autoantibodies (Freire de Carvalho et al., 2010; Lu et al., 2010; Molina et al., 1996). Similarly, RRMS affects women about twice as often as men, and overall male gender has also been associated with worse prognosis. Although some small studies reported that men with MS are prone to develop less inflammatory but more destructive lesions than women (Pozzilli et al., 2003), have less T2 and gadolinium(Gd)-enhancing lesions (Weatherby et al., 2000) but more T1 lesions (van Walderveen et al., 2001) and more brain atrophy (Antulov et al., 2009), gender-specific MRI differences have not been confirmed by large studies (Fazekas et al., 2009). However, gender appears to influence disability during the early phase of RRMS. When males are compared with females, they do not show differences at the age of RRMS onset, but females progress more slowly and thus are overall older when they reach a disability status scale of 3 (Leray et al., 2010). Since disability evolution in RRMS correlates with axonal injury and this in turn correlates with inflammation, all these data suggest that the nature of inflammation and/or a higher vulnerability of CNS tissue in RRMS males might underlie the worse prognosis in these patients. Higher circulating NETs levels 
might prime $T$ cells and other immune cell types as well as facilitate their migration through the blood brain barrier and, as a consequence, lead to more destructive neuroinflammatory processes in male RRMS patients.

In order to understand better the potential causes of NETosis in RRMS patients we have performed a systematic and in depth characterization of these patients. Our data show that high levels of NETs are not stable in serum, but fluctuate over time. While this might indicate an association with disease activity, which comes and goes in MS, we could not demonstrate a correlation between relapses and higher circulating NETs in serum. Regarding potential triggers, we could not either demonstrate an involvement of inflammation, infection, platelet nor endothelial activation. One important question to consider in the interpretation of these results is the stability and half-life of MPO/DNA complexes in serum. Different from free DNA that is easily digested by DNAses, MPO-DNA complexes appear to be much more stable, and consequently their presence may not always indicate recent NETosis. This open issue might be one of the reasons for our difficulties to identify triggers of NETs.

Further we have addressed a putative role of these complexes in RRMS pathogenesis and gender-specific differences by systematically characterizing circulating lymphocytes, DCs and monocytes. Unfortunately, the analysis of circulating lymphocytes and DCs did not provide information regarding a putative role of NETs in MS pathogenesis and neither regarding genderspecific differences. Unexpectedly, the analysis of circulating monocytes showed significant higher monocyte counts in whole blood of RRMS patients with higher circulating NETs and particularly in male patients. The phenotypic characterization of classical, intermediate and non-classical monocytes in these patients did not allow us to identify the monocyte population expanded, which suggests that we are either missing other monocyte populations or that our cell counter-based monocyte counts enumerate as monocytes another cell type with similar physical features. The identification and characterization of this cell population expanded in RRMS with high NETs requires further investigation. 
Finally, we also examined a putative link between NETs in serum and autoantibody production in RRMS patients. ds-DNA auto-antibodies and cANCA were not detected in any sample. Regarding ANA and pANCA, although the differences were not significant, the tendency to higher level in males might deserve further investigation. As mentioned above with respect to triggers of NETs, the lack of information concerning the exact time point of NETosis most likely hinders the search for a putative role of these complexes in RRMS pathogenesis.

The well documented role of NETs in the pathogenesis of some autoimmune diseases, their ability to modulate $T$ cell immunity and our previous observation that higher levels of NETs in serum characterize a subset of RRMS patients encouraged us to analyze in detail whether abnormally high levels of NETs may play a role in certain aspects of this $T$ cell-mediated autoimmune disease. As discussed above, the low frequency of RRMS patients with high levels of circulating NETs as well as the results from our characterization of these patients argue against a major role of NETosis in disease pathogenesis. However, our observation that individuals with high level of circulating NETs were significantly higher among RRMS patients than among $\mathrm{HC}$ or patients with other MS subforms, and more importantly that the frequency of males belonging to this subset was significantly higher than of females, suggests that NETosis might be responsible for some of the genderspecific differences in this disease and be involved in some aspects of MS pathogenesis such as opening of the blood brain barrier. Further investigation of gender-specific differences in NETosis using other methodological approaches might clarify its role in MS pathomechanisms and -heterogeneity. 


\section{Acknowledgment}

We thank all patients and healthy donors for their blood donations and the staff of the inims outpatient clinic and the staff of the Department for Transfusion Medicine, University Medical Centre Hamburg-Eppendorf for providing the samples. The inims was supported by the Gemeinnützige Hertie Stiftung, and this project was supported by the DFG grant SO 1029/1-1. 


\section{References}

Amulic, B., Cazalet, C., Hayes, G.L., Metzler, K.D., Zychlinsky, A., 2012. Neutrophil Function: From Mechanisms to Disease. Annu. Rev. Immunol. 30, 459-489.

Antulov, R., Weinstock-Guttman, B., Cox, J.L., Hussein, S., Durfee, J., Caiola, C., Dwyer, M.G., Bergsland, N., Abdelrahman, N., Stosic, M., Hojnacki, D., Munschauer, F.E., Miletic, D., Zivadinov, R., 2009. Gender-related differences in MS: a study of conventional and nonconventional MRI measures. Multiple Sclerosis 15, 345-354.

Bain, B.J., England, J.M., 1975a. Normal haematological values: sex difference in neutrophil count. Br Med J 1, 306-309.

Bain, B.J., England, J.M., 1975b. Variations in leucocyte count during menstrual cycle. Br Med J 2, 473-475.

Barrat, F.J., Meeker, T., Gregorio, J., Chan, J.H., Uematsu, S., Akira, S., Chang, B., Duramad, O., Coffman, R.L., 2005. Nucleic acids of mammalian origin can act as endogenous ligands for Toll-like receptors and may promote systemic lupus erythematosus. J Exp Med 202, 1131-1139.

Brinkmann, V., Reichard, U., Goosmann, C., Fauler, B., Uhlemann, Y., Weiss, D.S., Weinrauch, Y., Zychlinsky, A., 2004. Neutrophil extracellular traps kill bacteria. Science 303, 1532-1535.

Clark, S.R., Ma, A.C., Tavener, S.A., McDonald, B., Goodarzi, Z., Kelly, M.M., Patel, K.D., Chakrabarti, S., McAvoy, E., Sinclair, G.D., Keys, E.M., AllenVercoe, E., Devinney, R., Doig, C.J., Green, F.H., Kubes, P., 2007. Platelet TLR4 activates neutrophil extracellular traps to ensnare bacteria in septic blood. Nat Med 13, 463-469.

Diana, J., Simoni, Y., Furio, L., Agerberth, B., Barrat, F., Lehuen, A., 2013. Crosstalk between neutrophils, B-1a cells and plasmacytoid dendritic cells initiates autoimmune diabetes. Nature Medicine 19, 65-73.

Efrati, P., Presentey, B., Margalith, M., Rozenszajn, L., 1964. Leukocytes of Normal Pregnant Women. Obstet Gynecol 23, 429-432.

Fazekas, F., Enzinger, C., Wallner-Blazek, M., Ropele, S., Pluta-Fuerst, A., Fuchs, S., 2009. Gender differences in MRI studies on multiple sclerosis. Journal of Neurological Sciences 286, 28-30.

Freire de Carvalho, J., do Nascimento, A.P., Testagrossa, L.A., Toledo-Barros, R., Bonfa, E., 2010. Male gender results in more severe lupus nephitis. Reumatol Int 30, 1311-1315.

Fuchs, T.A., Abed, U., Goosmann, C., Hurwitz, R., Schulze, I., Wahn, V., Weinrauch, Y., Brinkmann, V., Zychlinsky, A., 2007. Novel cell death program leads to neutrophil extracellular traps. J Cell Biol 176, 231-241.

Fukazawa, T., Hamada, T., Kikuchi, S., Sasaki, H., Tashiro, K., Maguchi, S., 1996. Antineutrophil cytoplasmic antibodies and the optic-spinal form of multiple sclerosis in Japan. J Neurol Neurosurg Psychiatry 61, 203-204.

Garcia-Romo, G.S., Caielli, S., Vega, B., Connolly, J., Allantaz, F., Xu, Z., Punaro, M., Baisch, J., Guiducci, C., Coffman, R.L., Barrat, F.J., Banchereau, J., Pascual, V., 2011. Netting neutrophils are major inducers of type I IFN production in pediatric systemic lupus erythematosus. Sci Transl Med 3, $73 \mathrm{ra} 20$.

Granieri, E., Casetta, I., Tola, M.R., Ferrante, P., 2001. Multiple sclerosis: infectious hypothesis. Neurol Sci 22, 179-185. 
Gupta, A.K., Hasler, P., Holzgreve, W., Gebhardt, S., Hahn, S., 2005. Induction of neutrophil extracellular DNA lattices by placental microparticles and IL-8 and their presence in preeclampsia. Hum Immunol 66, 1146-1154.

Gupta, A.K., Joshi, M.B., Philippova, M., Erne, P., Hasler, P., Hahn, S., Resink, T.J., 2010. Activated endothelial cells induce neutrophil extracellular traps and are susceptible to NETosis-mediated cell death. FEBS Lett 584, 3193-3197.

Hampton, M.B., Kettle, A.J., Winterbourn, C.C., 1998. Inside the neutrophil phagosome: oxidants, myeloperoxidase, and bacterial killing. Blood 92, 30073017.

Kaplan, K.L., Owen, J., 1981. Plasma levels of beta-thromboglobulin and platelet factor 4 as indices of platelet activation in vivo. Blood 57, 199-202.

Kessenbrock, K., Krumbholz, M., Schonermarck, U., Back, W., Gross, W.L., Werb, Z., Grone, H.J., Brinkmann, V., Jenne, D.E., 2009. Netting neutrophils in autoimmune small-vessel vasculitis. Nat Med 15, 623-625.

Knight, J.S., Kaplan, M.J., 2012. Lupus neutrophils: "NET" gain in understanding lupus pathogenesis. Current Opinion in Reumatology 24, 441-450.

Lande, R., Ganguly, D., Facchinetti, V., Frasca, L., Conrad, C., Gregorio, J., Meller, S., Chamilos, G., Sebasigari, R., Riccieri, V., Bassett, R., Amuro, H., Fukuhara, S., Ito, T., Liu, Y.J., Gilliet, M., 2011. Neutrophils activate plasmacytoid dendritic cells by releasing self-DNA-peptide complexes in systemic lupus erythematosus. Sci Transl Med 3, 73ra19.

Lande, R., Gregorio, J., Facchinetti, V., Chatterjee, B., Wang, Y.H., Homey, B., Cao, W., Wang, Y.H., Su, B., Nestle, F.O., Zal, T., Mellman, I., Schroder, J.M., Liu, Y.J., Gilliet, M., 2007. Plasmacytoid dendritic cells sense self-DNA coupled with antimicrobial peptide. Nature 449, 564-569.

Lehrer, R.I., Ganz, T., 1999. Antimicrobial peptides in mammalian and insect host defence. Curr Opin Immunol 11, 23-27.

Leray, E., Yaouanq, J., Le Page, E., Coustans, M., Laplaud, D., Oger, J.I., G., E., 2010. Evidence for a two-stage disability progression in multiple sclerosis. Brain 133, 1900-1913.

Logters, T., Margraf, S., Altrichter, J., Cinatl, J., Mitzner, S., Windolf, J., Scholz, M., 2009. The clinical value of neutrophil extracellular traps. Med Microbiol Immunol 198, 211-219.

Lu, L.-J., Wallace, D.J., Ishimori, M.L., Scofield, R.H., Weisman, M.H., 2010. Review: Male systemic lupus erythematosus: a review of sex disparities in this disease. Lupus 19, 119-129.

Lund, B.T., Ashikian, N., Ta, H.Q., Chakryan, Y., Manoukian, K., Groshen, S., Gilmore, W., Cheema, G.S., Stohl, W., Burnett, M.E., Ko, D., Kachuck, N.J., Weiner, L.P., 2004. Increased CXCL8 (IL-8) expression in Multiple Sclerosis. J Neuroimmunol 155, 161-171.

Martin, G.S., Mannino, D.M., Eaton, S., Moss, M., 2003. The epidemiology of sepsis in the United States from 1979 through 2000. N Engl J Med 348, 1546-1554.

Means, T.K., Latz, E., Hayashi, F., Murali, M.R., Golenbock, D.T., Luster, A.D., 2005. Human lupus autoantibody-DNA complexes activate DCs through cooperation of CD32 and TLR9. J Clin Invest 115, 407-417.

Minagar, A., Jy, W., Jimenez, J.J., Sheremata, W.A., Mauro, L.M., Mao, W.W., Horstman, L.L., Ahn, Y.S., 2001. Elevated plasma endothelial microparticles in multiple sclerosis. Neurology 56, 1319-1324.

Molina, J.F., Drenkard, C., J., M., H., C.M., Uribe, O., M., A.J., Gomez, L.J., Felipe, O., Ramirez, L.A., Alarcon-Segovia, D., 1996. Systemic lupus erythematosus 
in males. A study of 107 Latin American patients. Medicine (Baltimore) 75, 124-130.

Molloy, E.J., O'Neill, A.J., Grantham, J.J., Sheridan-Pereira, M., Fitzpatrick, J.M., Webb, D.W., Watson, R.W., 2003. Sex-specific alterations in neutrophil apoptosis: the role of estradiol and progesterone. Blood 102, 2653-2659.

Naegele, N., Tillack, K., Reinhardt, S., Schippling, S., Martin, R., Sospedra, M., 2012. Neutrophils in Multiple Sclerosis are characterized by a primed phenotype. Journal of Neuroimmunology 242, 60-71.

Polman, C.H., Reingold, S.C., Edan, G., Filippi, M., Hartung, H.P., Kappos, L., Lublin, F.D., Metz, L.M., McFarland, H.F., O'Connor, P.W., SandbergWollheim, M., Thompson, A.J., Weinshenker, B.G., Wolinsky, J.S., 2005. Diagnostic criteria for multiple sclerosis: 2005 revisions to the "McDonald Criteria". Ann Neurol 58, 840-846.

Pozzilli, C., Tomassini, V., Marinelli, F., Paolillo, A., Gasperini, C., Bastianello, S., 2003. 'Gender gap' in multiple sclerosis: magnetic resonance imaging evidence. . Eur J Neurol 10, 95-97.

Sangaletti, S., Tripodo, C., Chiodoni, C., Guarnotta, C., Cappetti, B., Casalini, P., Piconese, S., Parenza, M., Guiducci, C., Vitali, C., Colombo, M.P., 2012. Neutrophil extracellular traps mediate transfer of cytoplasmic neutrophil antigens to myeloid dendritic cells toward ANCA induction and associated autoimmunity. Blood 120, 3007-3018.

Schroder, J., Kahlke, V., Staubach, K.H., Zabel, P., Stuber, F., 1998. Gender differences in human sepsis. Arch Surg 133, 1200-1205.

Segal, A.W., 2005. How neutrophils kill microbes. Annu Rev Immunol 23, 197-223.

Sheremata, W.A., Jy, W., Horstman, L.L., Ahn, Y.S., Alexander, J.S., Minagar, A., 2008. Evidence of platelet activation in multiple sclerosis. J

Neuroinflammation 5, 27.

Sospedra, M., Martin, R., 2005. Immunology of multiple sclerosis. Annu Rev Immunol 23, 683-747.

Stuckey, J.A., St Charles, R., Edwards, B.F., 1992. A model of the platelet factor 4 complex with heparin. Proteins 14, 277-287.

Tillack, K., Breiden, P., Martin, R., Sospedra, M., 2012. T Lymphocyte Priming by Neutrophil Extracellular Traps Links Innate and Adaptive Immune Responses. Journal of Immunology 188, 3150.

Urban, C.F., Ermert, D., Schmid, M., Abu-Abed, U., Goosmann, C., Nacken, W., Brinkmann, V., Jungblut, P.R., Zychlinsky, A., 2009. Neutrophil extracellular traps contain calprotectin, a cytosolic protein complex involved in host defense against Candida albicans. PLoS Pathog 5, e1000639.

van Walderveen, M.A., Lycklama, A., Nijeholt, G.J., Ader, H.J., Jongen, P.J., Polman, C.H., Castelijns, J.A., Barkhof, F., 2001. Hypointense lesions on T1weighted spin-echo magnetic resonance imaging: relation to clinical characteristics in subgroups of patients with multiple sclerosis. . Arch Neurol 58, 76-91.

Vestweber, D., 2008. VE-cadherin: the major endothelial adhesion molecule controlling cellular junctions and blood vessel formation. Arterioscler Thromb Vasc Biol 28, 223-232.

Weatherby, S.J., Mann, C.L., Davies, M.B., Fryer, A.A., Haq, N., Strange, R.C., Hawkins, C.P., 2000. A pilot study of the relationship between gadoliniumenhancing lesions, gender effect and polymorphisms of antioxidant enzymes in multiple sclerosis. . J Neurol 247. 
Weiss, J., Kao, L., Victor, M., Elsbach, P., 1985. Oxygen-independent intracellular and oxygen-dependent extracellular killing of Escherichia coli S15 by human polymorphonuclear leukocytes. J Clin Invest 76, 206-212. 


\section{Figure legends}

Figure 1. Gender differences in circulating levels of NETs in serum of RRMS patients. A) Quantification of MPO-DNA complexes in serum samples of HC $(n=40)$, CIS $(n=126)$, RRMS $(n=168)$, PPMS $(n=21)$, OIND $(n=5)$ and OND $(n=10)$ patients. An arbitrary threshold of $O D>0.3$ to differentiate samples with high MPO-DNA complexes is shown (dotted line). Percentage of donors/patients with OD $>0.3$, the mean OD as measured by capture ELISA and the statistical significance are shown. $\left({ }^{*} p<0.05\right)$. B) Quantification of MPO-DNA complexes in CSF samples from CIS $(n=20), \operatorname{RRMS~}(n=21)$, PPMS $(n=6)$, OIND $(n=5)$ and OND $(n=10)$ patients. C) Correlation between age and MPO-DNA complexes in all serum samples. D) Quantification of MPO-DNA complexes in serum samples of all females $(n=225)$ and males $(n=149)$, of HC females $(n=20)$ and males $(n=20)$, of CIS females $(n=78)$ and males $(n=50)$, of RRMS females $(n=107)$ and males $(n=61)$ and of PPMS females $(n=10)$ and males $(n=9)$. Percentage of donors/patients with OD > 0.3 , the mean optical density and statistical significance are shown. (* $\left.\mathrm{p}=0.0113,{ }^{* *} \mathrm{p}=0.0058\right)$.

Figure 2. Gender differences in NETs formation and degradation. A) Quantification of MPO-DNA complexes released in vitro by purified neutrophils from female $(n=33)$ and male $(n=22) H C$ and female $(n=20)$ and male $(n=8)$ RRMS patients after stimulation with $25 \mathrm{nM}$ PMA. The mean stimulation index $(\mathrm{SI})$ is shown. ( $\mathrm{SI}=\mathrm{OD}$ measured by capture ELISA in stimulated neutrophils / OD in unstimulated neutrophils. B) Quantification of MPO-DNA complexes in serum samples from pre-selected groups of female and male RRMS patients and CIS patients each group containing a equal number of individuals with high (OD $>0.3$, RRMS female $n=9$ and male $n=9$, CIS female $n=7$, males $=7$ ) or low (OD $<0.2$, RRMS female $n=9$ and male $n=9$, CIS female $n=6$, males $=8$ ) levels of MPO-DNA complexes (upper graphs). dsDNA ( $\mathrm{ng} / \mathrm{ml}$ ) quantified using Picogreen in the same groups of female and male patients (lower graphs). Mean and statistical significance are shown. ( $\left.{ }^{* *} \mathrm{p}=0.0046\right)$. C) dsDNA ( $\left.\mathrm{ng} / \mathrm{ml}\right)$ quantified using Picogreen in the same groups of female and male patients with high $(O D>0.3)$ and low 
$(\mathrm{OD}<0.2)$ MPO DNA complexes. Mean and statistical significance are shown. $\left({ }^{* *} p<0.01\right)$.

Figure 3. Circulating NETs in serum and disease activity. A) Quantification of MPO-DNA complexes in serum samples of 8 RRMS patients (4 females/4 males) obtained at different time points. B) Quantification of MPO-DNA complexes in serum samples of CIS females ( $n=40$ remission and 38 relapse), CIS males ( $n=29$ remission and 19 relapse), RRMS females ( $n=63$ remission and 43 relapse) and RRMS males ( $n=36$ remission and 26 relapse) C) Quantification of MPO-DNA complexes in serum samples from female (upper graphs) and male (lower graphs) RRMS patients for whose paired samples in remission and relapse were available. The left graphs shows patients with higher MPO-DNA complexes in relapse than in remission, while the right graph shows patients with higher MPO-DNA complexes in remission than in relapse. An arbitrary threshold of $O D>0.3$ to differentiate samples with high MPO-DNA complexes is shown in all graphs (dotted line).

Figure 4. Putative triggers of NET formation in RRMS patients. A) IL-8, CRP, IL-6, PF4, beta-TG and sVE cadherin quantification in serum samples of pre-selected groups of female (upper graphs) and male (lower graphs) RRMS patients with high ( $O D>0.3$, female $n=9$, male $n=9$ ) and low $(O D<0.2$, female $n=9$, male $n=9$ ) MPO-DNA complexes. The mean is shown in all graphs.

Figure 5. Circulating NETs and peripheral blood lymphocytes. A) Absolute peripheral blood counts of lymphocytes obtained using a coulter counter in female and male HC and RRMS patients (RRMS females $n=35$, males $n=20 ; H C$ females $n=25$, males $n=15$ ). B) Absolute peripheral blood lymphocyte counts in female and male RRMS patients with high (OD > 3; females $n=12$, males $n=9)$ and low $(O D<0.2$; females $n=13$, males $n=10$ ) MPO-DNA complexes in serum. Mean \pm SEM are shown. Frequency (\%) determined by flow cytometry of $\mathrm{CD}^{+}{ }^{+} \mathrm{CD} 8^{+}$and $\mathrm{CD} 3^{+} \mathrm{CD} 8^{+}$IFNy-producing $\mathrm{T}$ cells $(\mathrm{CD} 8+\mathrm{IFNY}+)(\mathrm{C})$, and $\mathrm{CD}^{+} \mathrm{CD} 4^{+}$, Th1 $\left(\mathrm{CD}^{+} \mathrm{CD}^{+} \mathrm{IFNY} \mathrm{IL}^{+} \mathrm{IL}^{-} \mathrm{IL}-17 \mathrm{~A}^{-}\right)$, Th2 ( $\left.\mathrm{CD}^{+} \mathrm{CD}^{+}{ }^{+} \mathrm{IFNY} \mathrm{IL}^{-} 4^{+}\right)$and Th17 $\left(\mathrm{CD}^{+} \mathrm{CD}^{+}{ }^{+} \mathrm{IFNy} \mathrm{IL}^{-}-17 \mathrm{~A}^{+}\right) \mathrm{T}$ cells (D) in PBMCs of female and male RRMS patients with high $(O D>0.3$, female 
$\mathrm{n}=8$, male $\mathrm{n}=8$ ) and low (OD $<0.2$, female $\mathrm{n}=8$, male $\mathrm{n}=8$ ) MPO-DNA complexes in serum. Mean is shown in all graphs.

Figure 6. Circulating NETs and peripheral blood dendritic cells. Gating strategy used to identify mDCs type $1\left(\mathrm{CD} 1 \mathrm{c}^{+}\right)$, mDCs type $2\left(\mathrm{CD} 141^{+}\right)$and

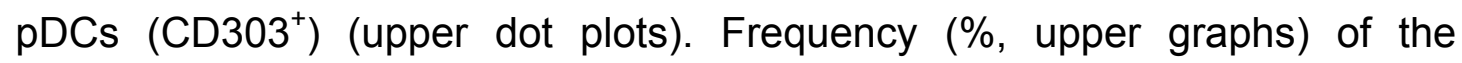
different DCs and CD40 and CD86 expression (MedFI, lower graphs) in PBMCs of female and male RRMS patients with high $(O D>0.3$; females $n=15$, males $n=13$ ) and low (OD < 0.2; females $n=14$, males $n=13$ ) MPO-DNA complexes in serum. Mean is shown in all graphs.

Figure 7. Circulating NETs and peripheral blood monocytes. A) Absolute peripheral blood counts of monocytes obtained using a coulter counter in female and male $\mathrm{HC}$ and RRMS patients (RRMS females $n=35$, males $n=20$; $\mathrm{HC}$ females $n=25$, males $n=15$ ). B) Absolute peripheral blood monocyte counts in female and male RRMS patients with high (OD > 3; females $n=12$, males $n=9$ ) and low (OD < 0.2; females $n=13$, males $n=10$ ) MPO-DNA complexes in serum. Mean \pm SEM and statistical significance are shown. $\left({ }^{*} p=0.0124 ;{ }^{* *} p<0.001\right)$. C) Frequency (\%, upper graphs) of classical, intermediate and non-classical monocytes and CD40 expression (MedFI, lower graphs) in PBMCs of female and male RRMS patients with high (OD > 0.3; females $n=10$, males $n=7$ ) and low ( $O D<0.2$; females $n=9$, males $n=7$ ) MPO-DNA complexes in serum. Mean is shown in all graphs. 
Table 1. Demographic characteristics of MS patients and controls.

\begin{tabular}{|l|c|c|c|c|c|}
\hline Group & $\mathbf{n}$ & $\mathbf{F}$ & $\mathbf{M}$ & $\mathbf{F : M}$ & Mean Age $\mathbf{\text { SD }}$ \\
\hline CIS & 126 & 78 & 48 & 1.6 & $33.9 \pm 8.8$ \\
\hline - CIS remission & 76 & 48 & 28 & 1.7 & $35 \pm 9.4$ \\
\hline - CIS relapse & 50 & 30 & 20 & 1.5 & $32.3 \pm 7.6$ \\
\hline RRMS & 168 & 106 & 62 & 1.7 & $36.5 \pm 7.9$ \\
\hline - RRMS remission & 99 & 63 & 36 & 1.7 & $37.1 \pm 7.5$ \\
\hline - RRMS relapse & 69 & 43 & 26 & 1.6 & $35.7 \pm 8.5$ \\
\hline PPMS & 21 & 11 & 10 & 1.1 & $39.3 \pm 6.6$ \\
\hline OIND & 5 & 3 & 2 & 1.5 & $47.8 \pm 20.5$ \\
\hline OND & 10 & 5 & 5 & 1 & $45.3 \pm 11$ \\
\hline HC & 40 & 20 & 20 & 1 & $32.2 \pm 7.3$ \\
\hline
\end{tabular}


Table 2. Autoantibodies in female and male RRMS patients with low and high MPO-DNA complexes in serum

\begin{tabular}{|c|c|c|c|c|c|}
\hline GENDER & $\begin{array}{l}\text { OD (MPO- } \\
\text { DNA) }\end{array}$ & ANA & cANCA & pANCA & Anti-dsDNA \\
\hline \multicolumn{6}{|l|}{ FEMALES } \\
\hline & 0,12 & neg & neg & neg & neg \\
\hline & 0,125 & neg & neg & neg & neg \\
\hline & 0,187 & pos $(1 / 320)$ & neg & neg & neg \\
\hline & 0,11 & pos $(1 / 1000)$ & neg & neg & neg \\
\hline & 0,126 & neg & neg & neg & neg \\
\hline & 0,135 & neg & neg & neg & neg \\
\hline \multicolumn{6}{|l|}{ MALES } \\
\hline & 0,121 & $\operatorname{pos}(1 / 320)$ & neg & pos (IgG 1/10) & neg \\
\hline & 0,131 & neg & neg & neg & neg \\
\hline & 0,155 & pos $(1 / 1000)$ & neg & $\operatorname{pos}(\lg G 1 / 10)$ & neg \\
\hline & 0,21 & pos $(1 / 1000)$ & neg & neg & neg \\
\hline \multicolumn{6}{|l|}{ 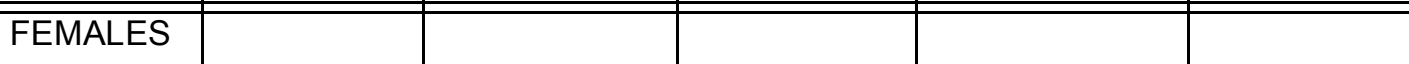 } \\
\hline & 0,413 & neg & neg & neg & neg \\
\hline & 0,477 & neg & neg & neg & neg \\
\hline & 0,779 & neg & neg & neg & neg \\
\hline & 0,901 & neg & neg & neg & neg \\
\hline & 1,291 & neg & neg & pos (IgG 1/10) & neg \\
\hline & 1,439 & neg & neg & neg & neg \\
\hline \multicolumn{6}{|l|}{ MALES } \\
\hline & 0,451 & neg & neg & neg & neg \\
\hline & 0,491 & neg & neg & neg & neg \\
\hline & 0,569 & pos $(1 / 320)$ & neg & neg & neg \\
\hline & 0,782 & pos $(1 / 320)$ & neg & neg & neg \\
\hline & 2,143 & neg & neg & $\operatorname{pos}(\operatorname{lgG} 1 / 10)$ & neg \\
\hline
\end{tabular}


A)

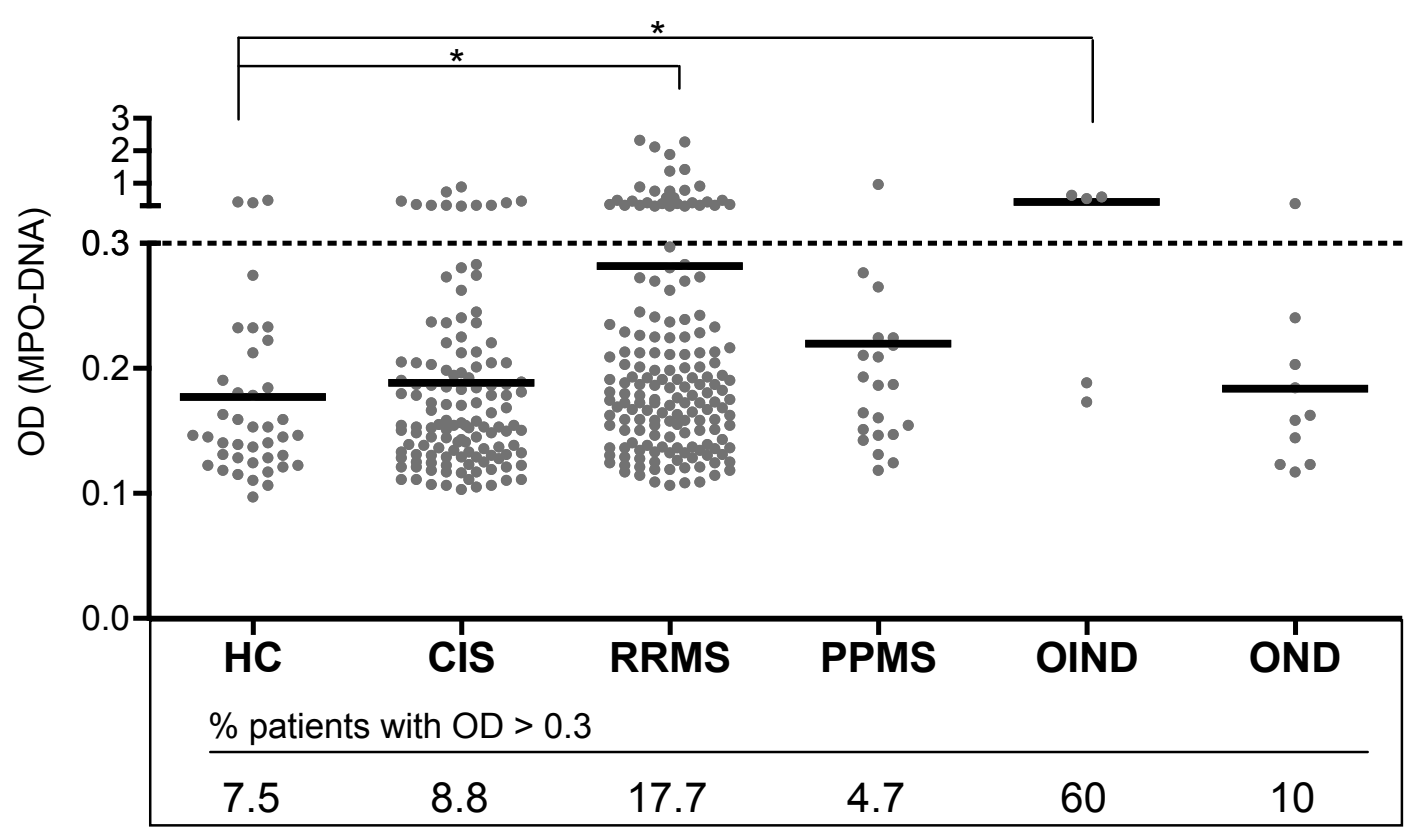

B)

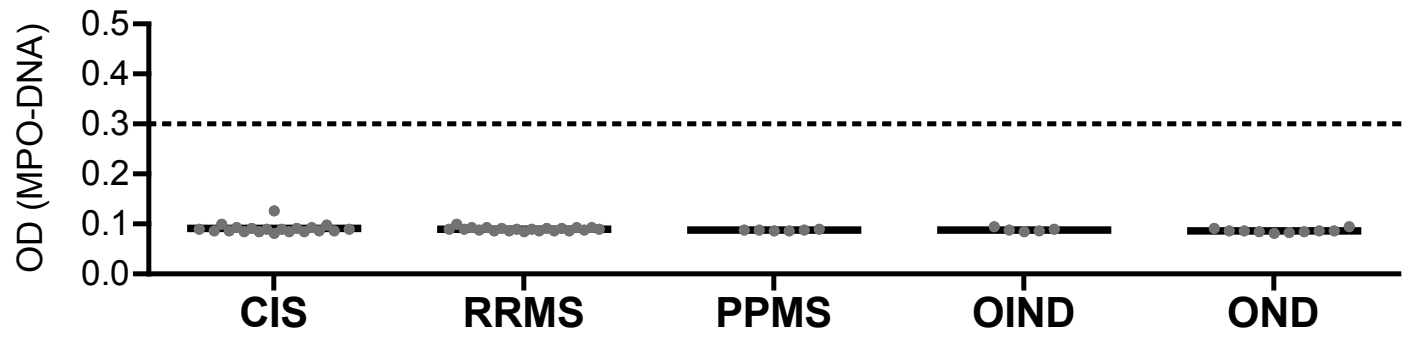

C)

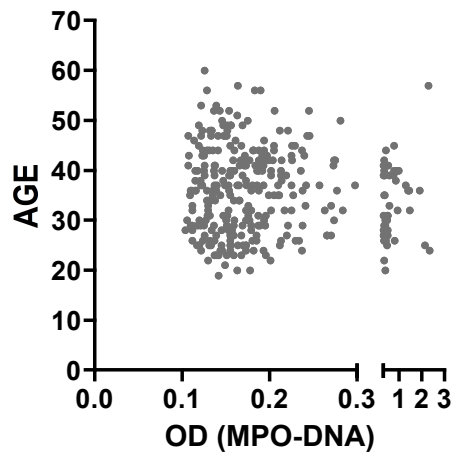

CIS

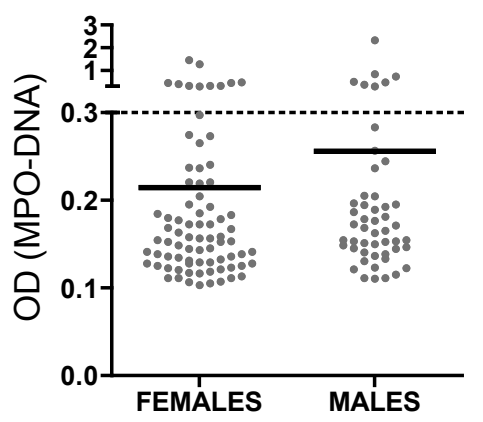

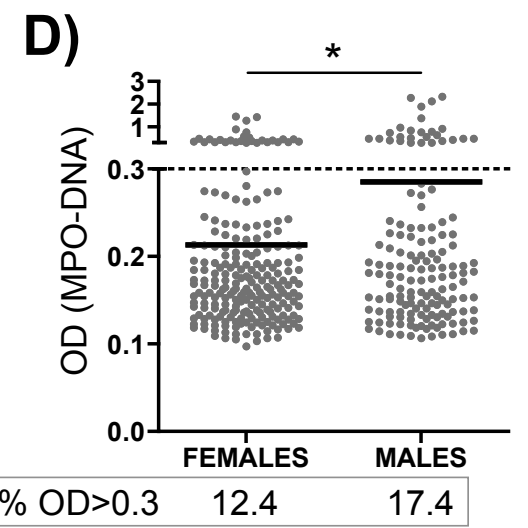

RRMS

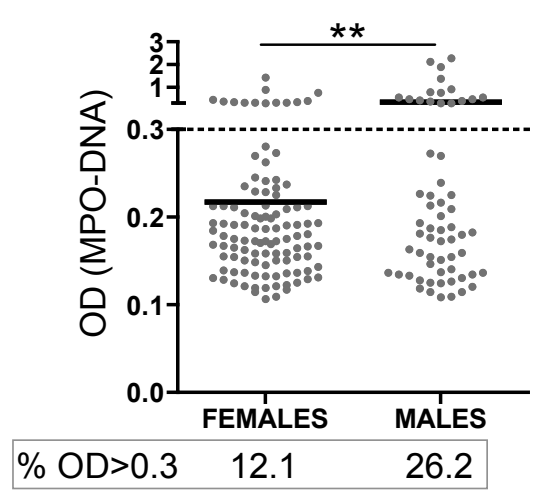

Figure 1 
Figure 2

A)

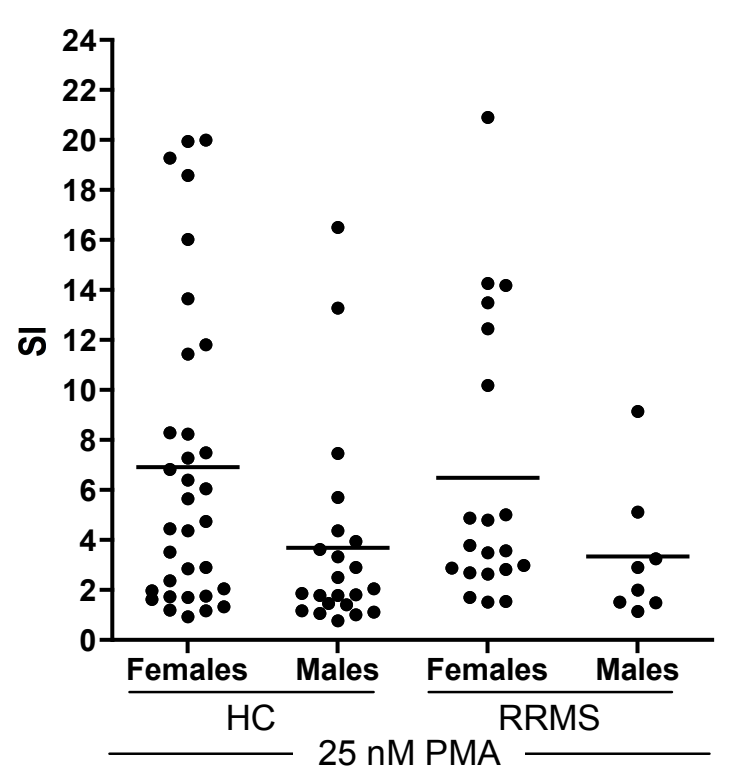

B)
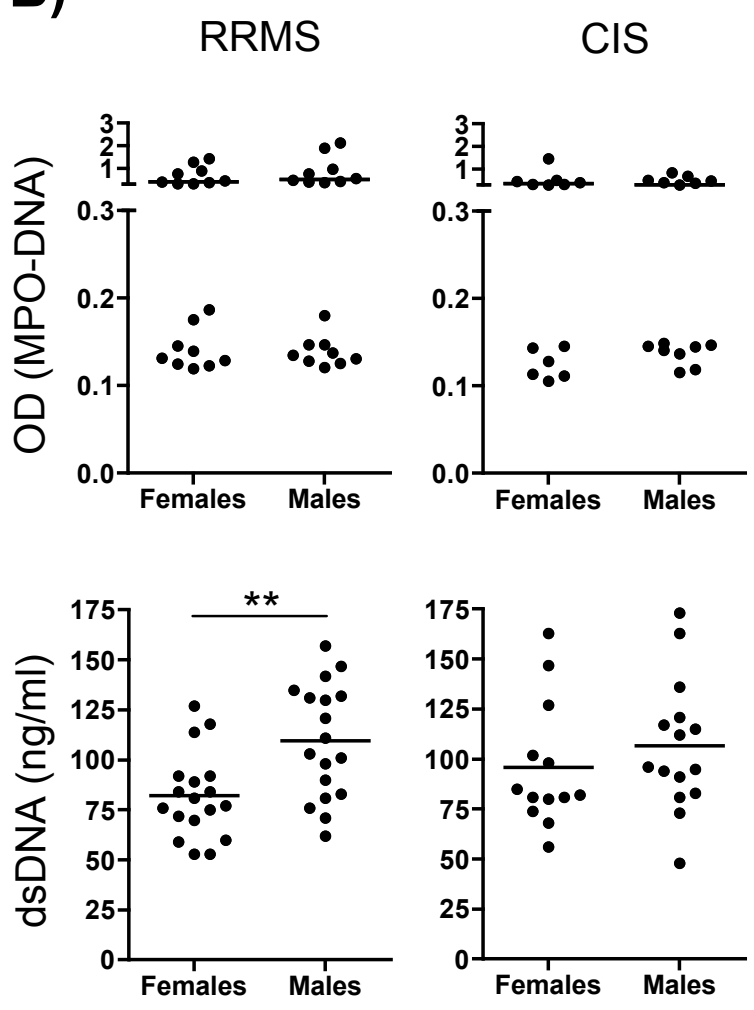

C)

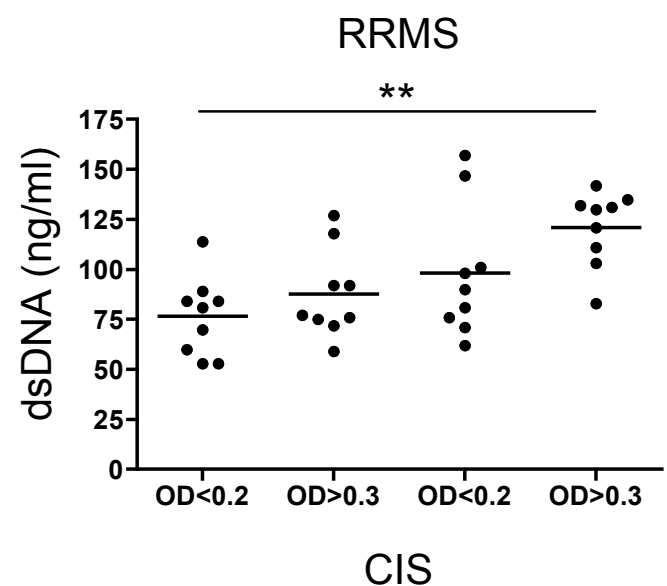


A)
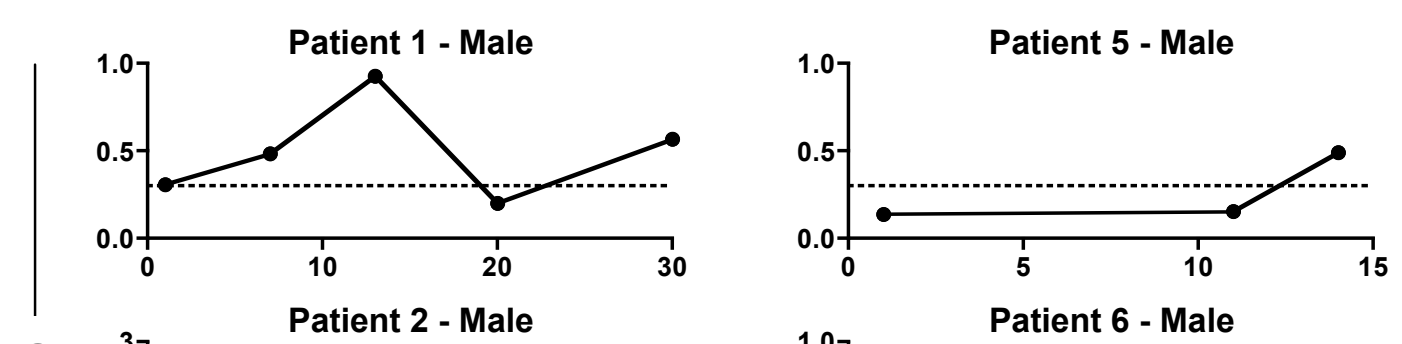

Figure 3
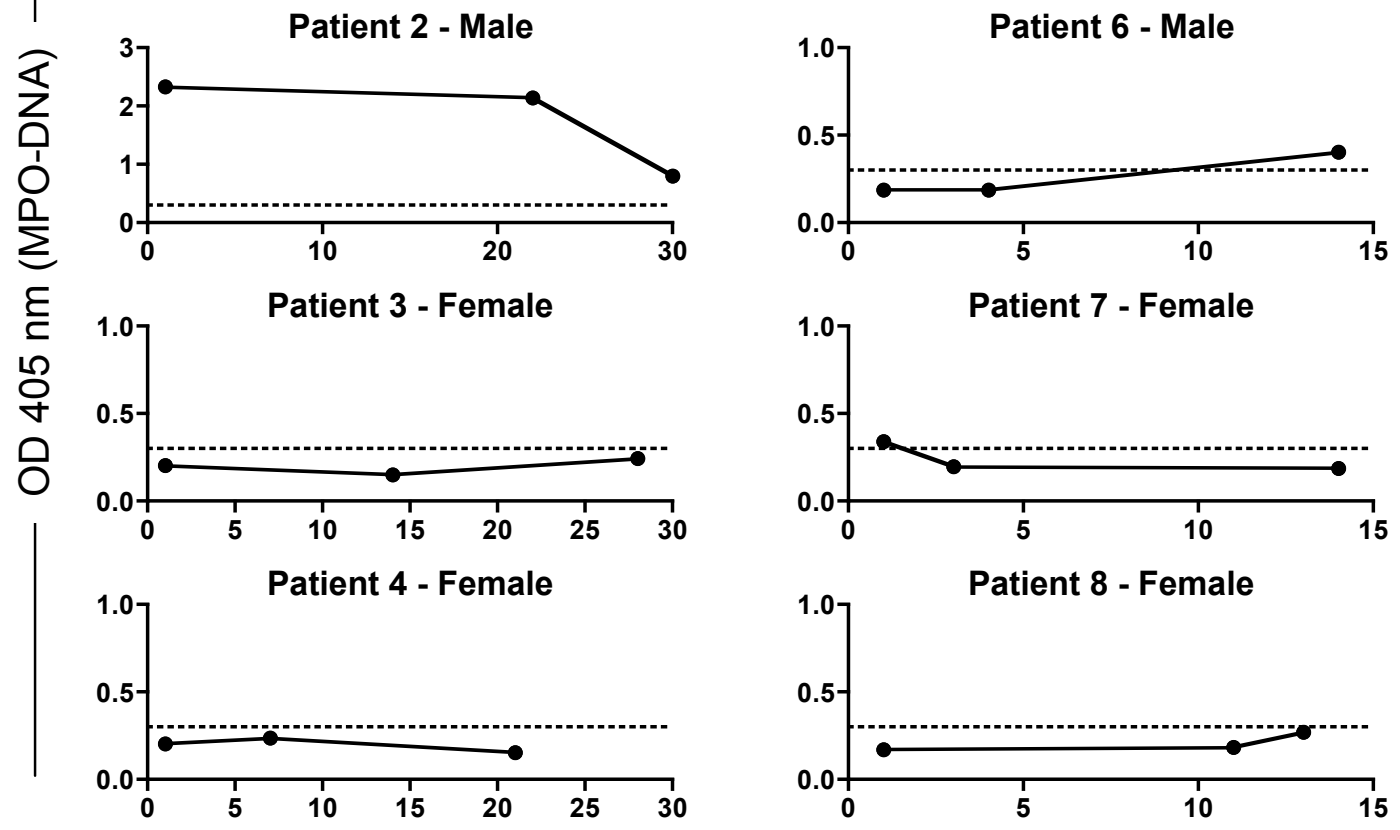

Months

B)

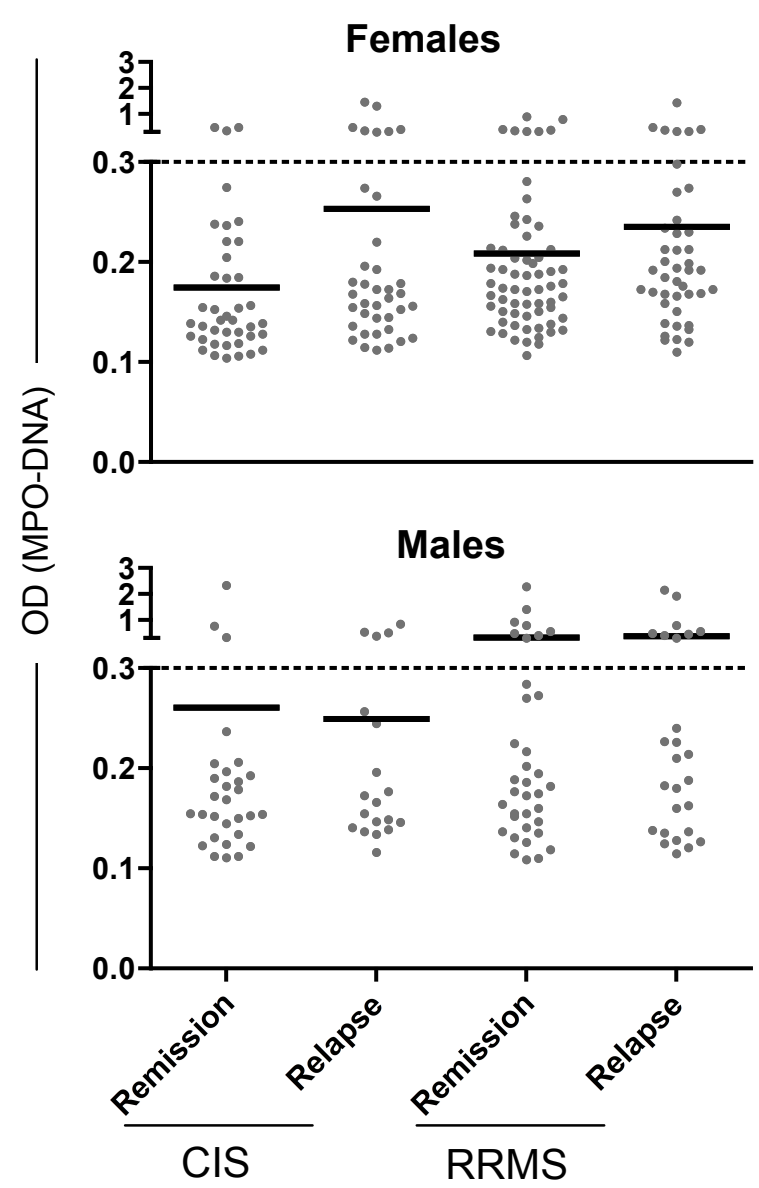

C)

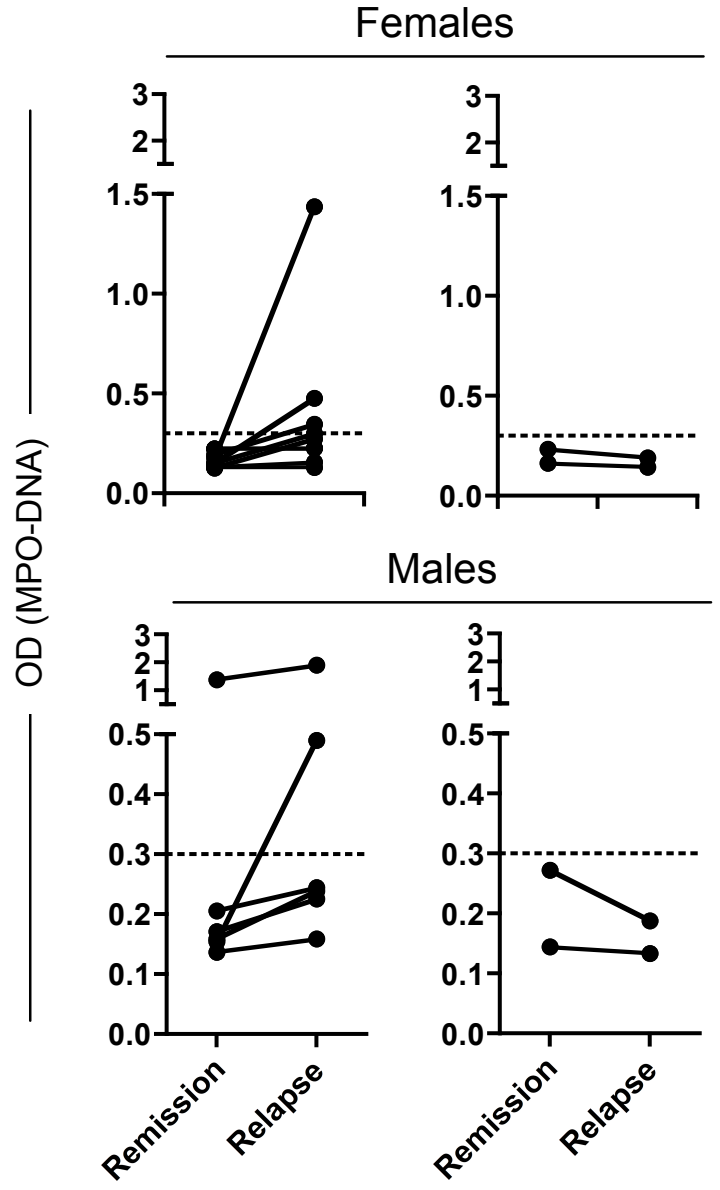


Figure 4
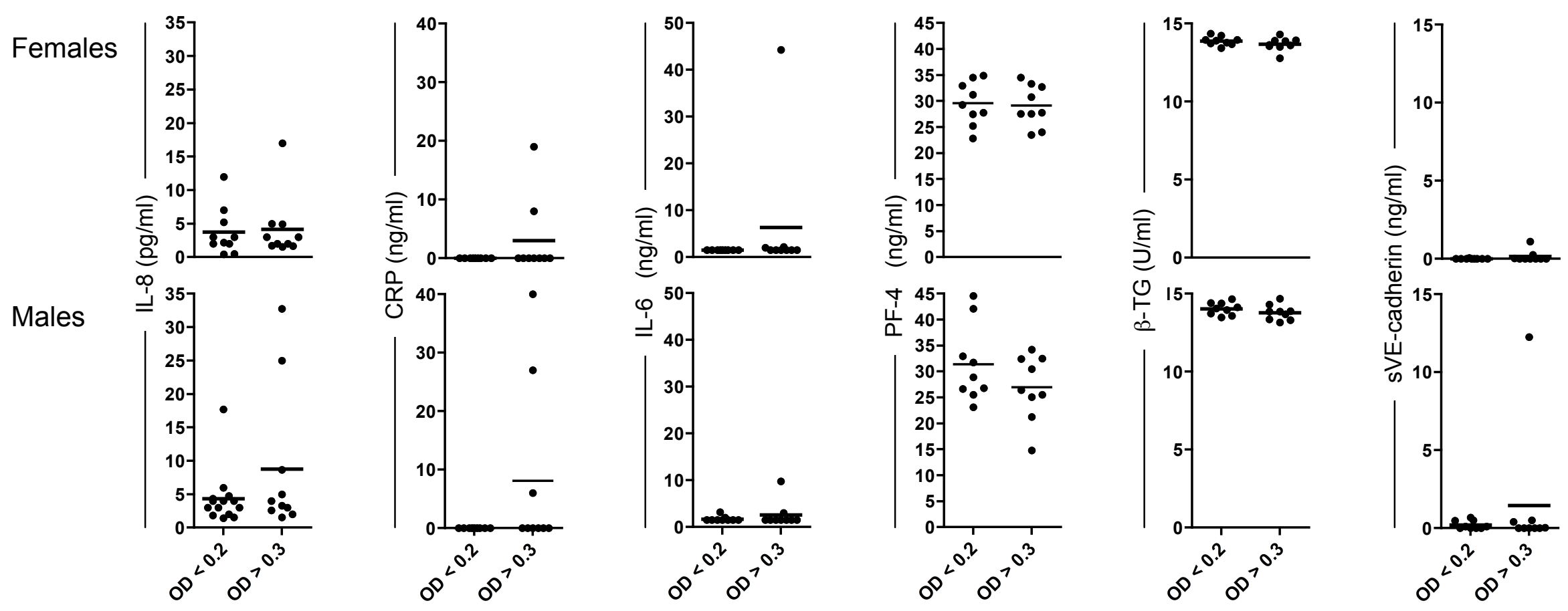
A)

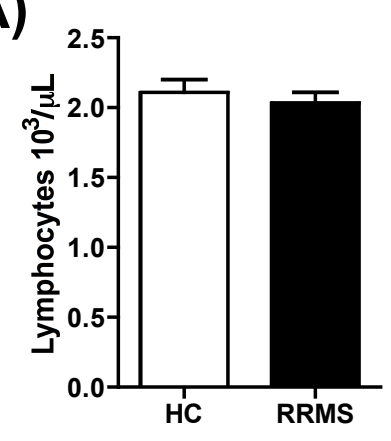

C)

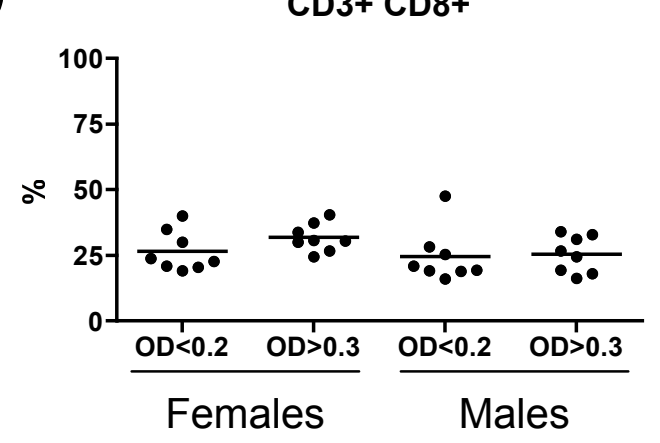

CD3+ CD8+ IFN $\gamma+$

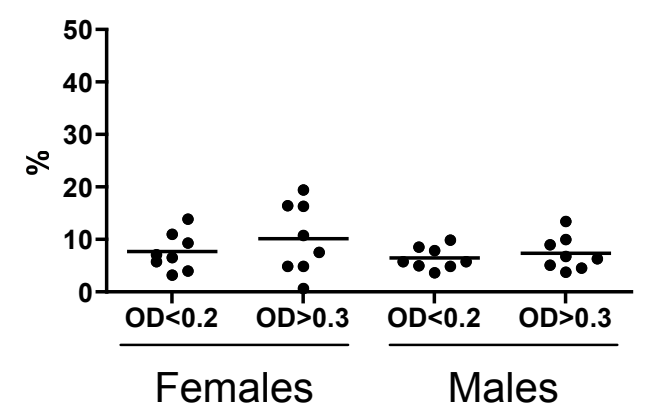

B)

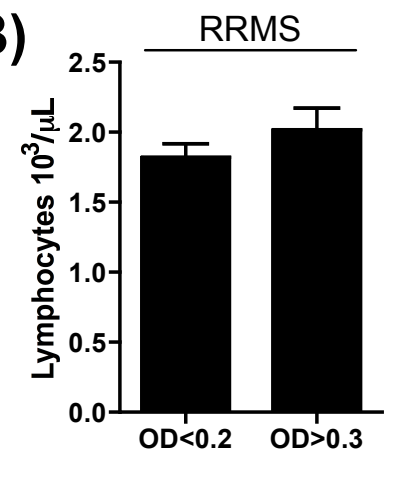

$\frac{\text { Females }}{\text { RRMS }}$

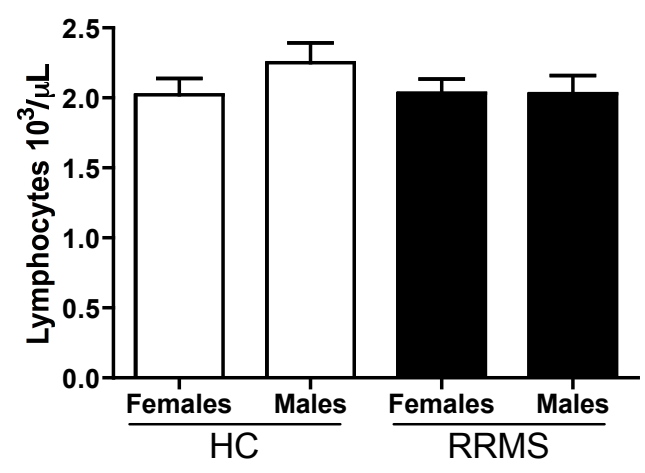

D)

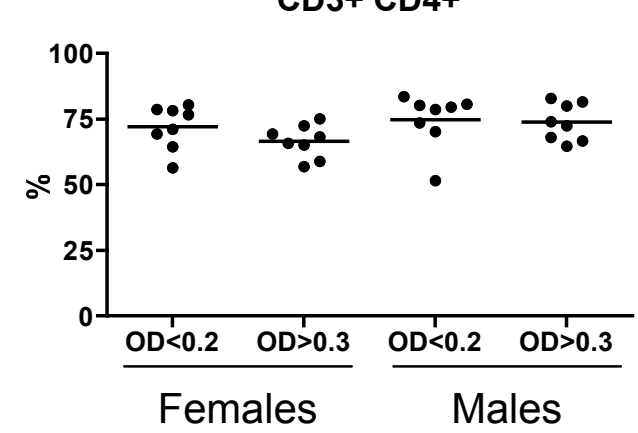

CD3+ CD4+ IFN ${ }^{+}$

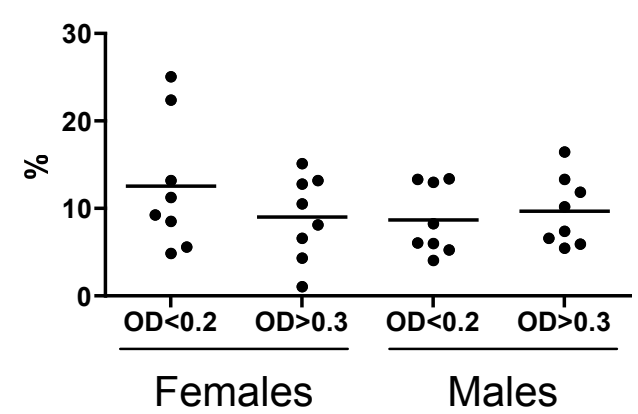

RRMS

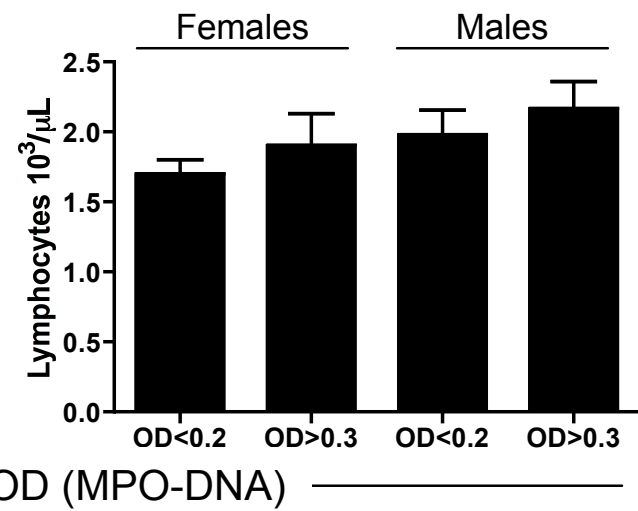

CD3+ CD4+ IL4+

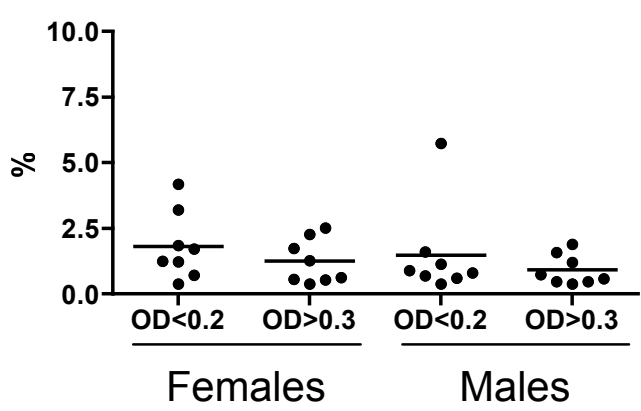

CD3+ CD4+ IL17+

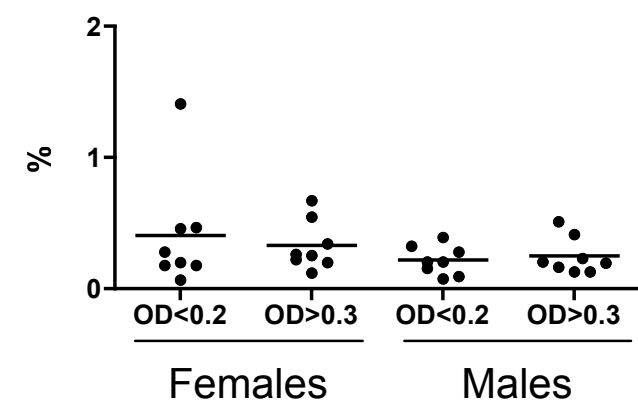

OD (MPO-DNA) 


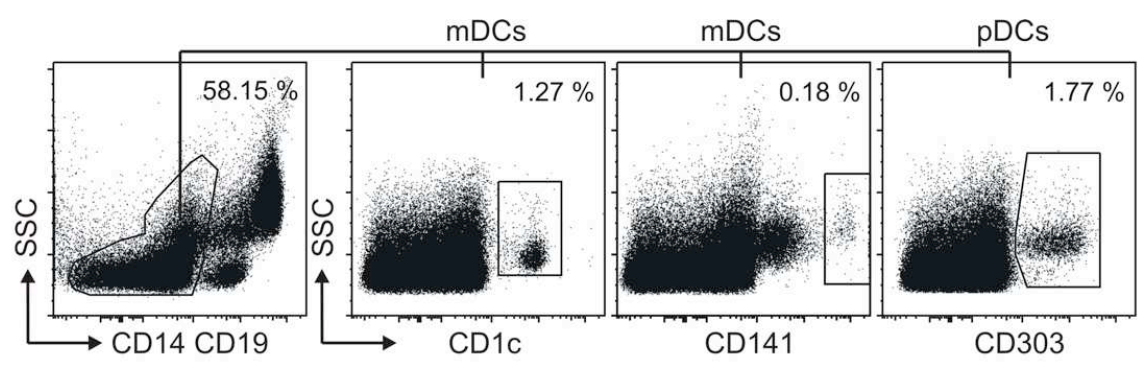

Figure 6
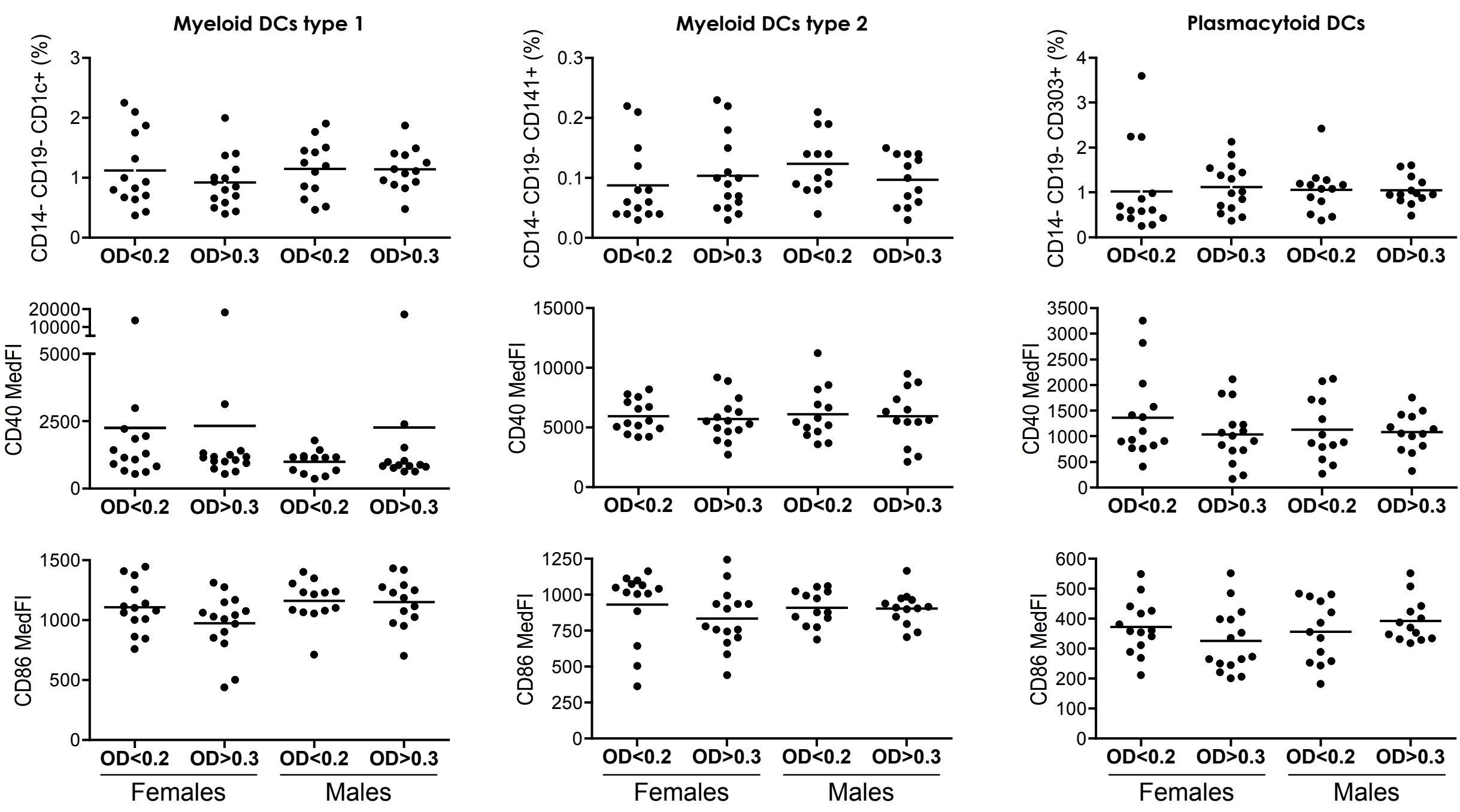
A)
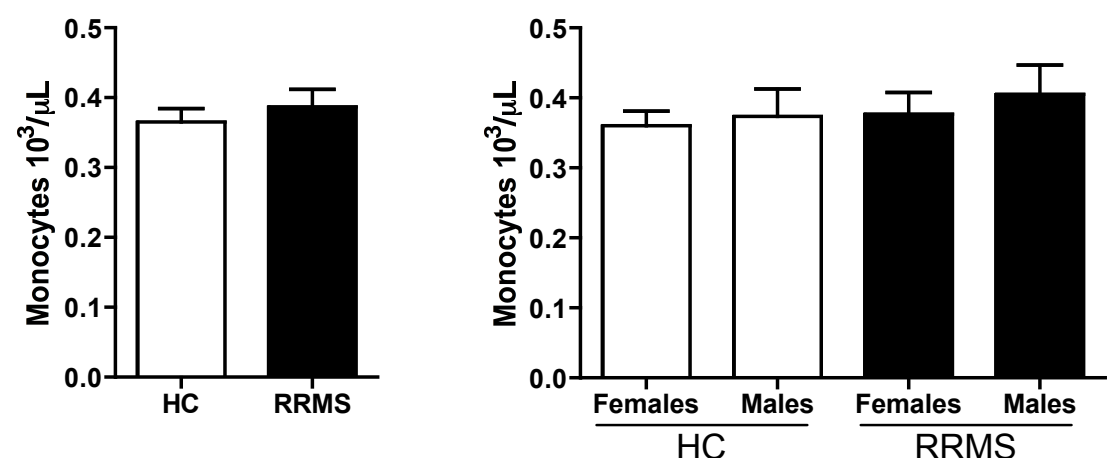

B)

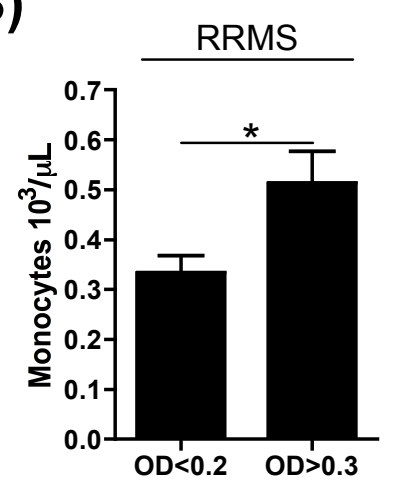

RRMS

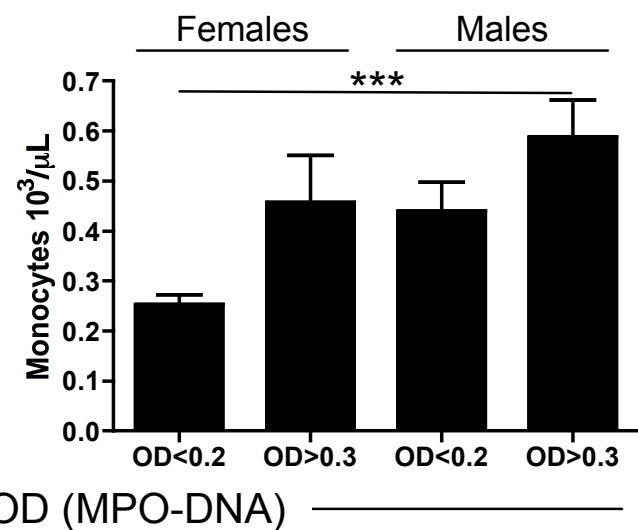

C)

Classical Monocytes
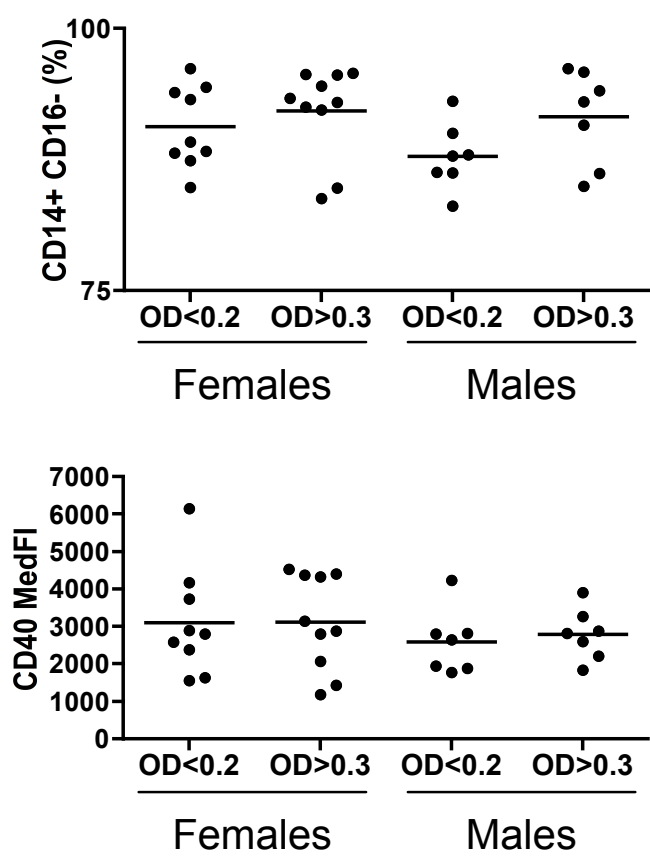

Intermediate Monocytes
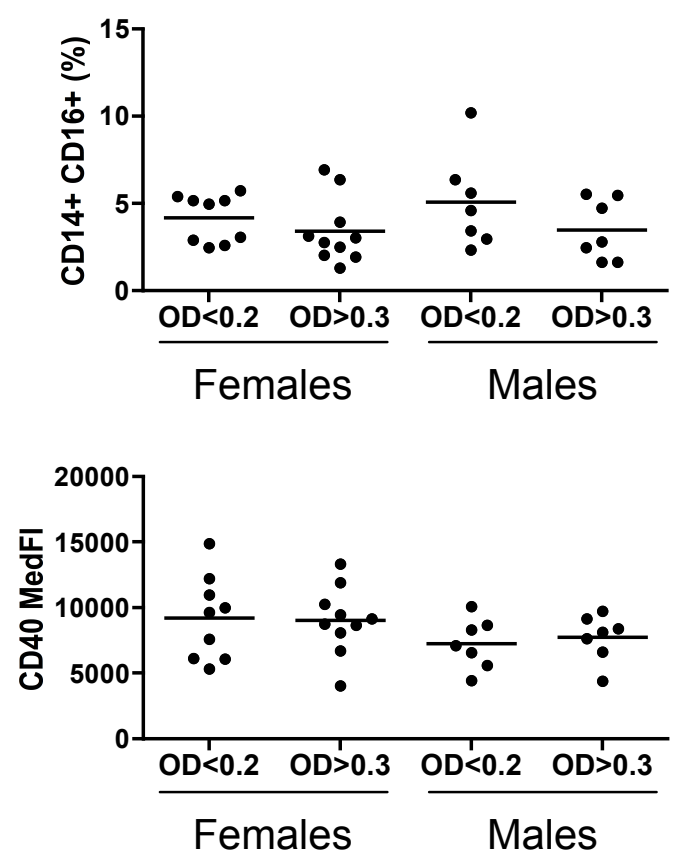

Non-classical Monocytes

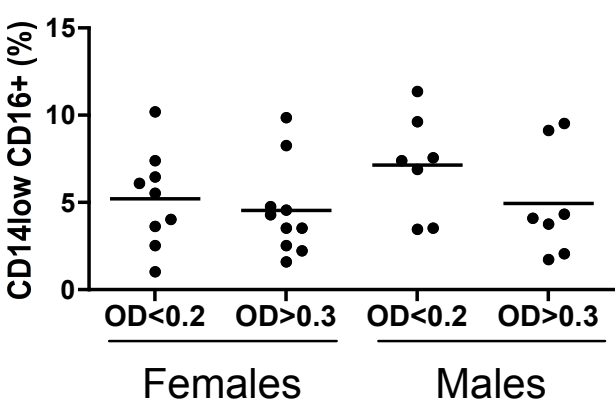

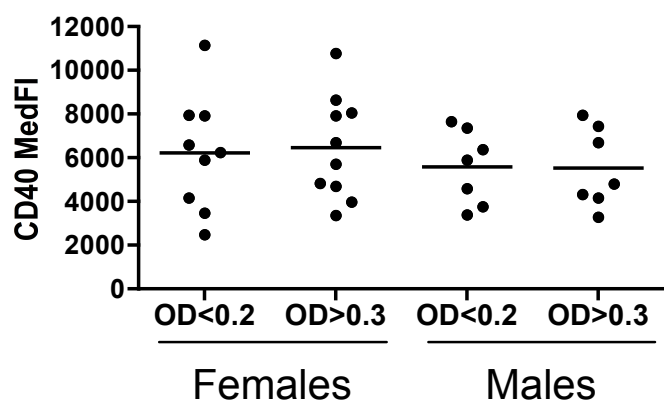


Supplementary Table-1. Diagnose of patients with inflammatory neurological diseases other than MS (OIND) and with non-inflammatory neurological diseases (OND)

\section{Conditi Diagnose}

on

OIND (Autoimmune) uveomeningeal syndrome

OIND Idiopathic trigeminal neuralgia (left-sided)

OIND Neuroborreliosis

OIND Neuro-Behçet's disease

OIND Paraneoplastic mixed axonal-demyelinating sensory-motor neuropathy (due to adenocarcinoma of the gastric cardia)

OND Shoulder pain of unknown etiology

OND Optical atrophy (suspected Leber's hereditary optic neuropathy)

OND Chronic musculoskeletal pain of unknown etiology (suspected psychosomatic presentations)

OND Muscle atrophy of unknown etiology

OND Hypokinetic-rigid idiopathic Parkinson's disease

OND Tinnitus of unknown etiology

OND Monoparesis of the right hand, most likely due to cerebral ischemia (underlying severe cerebral microangiopathy)

OND Suspected myoclonic epilepsy with ragged red fiber (MERRF) syndrome

OND distal paraesthesia of unknow etiology

OND migraine with aura 
Supplementary Figure 1

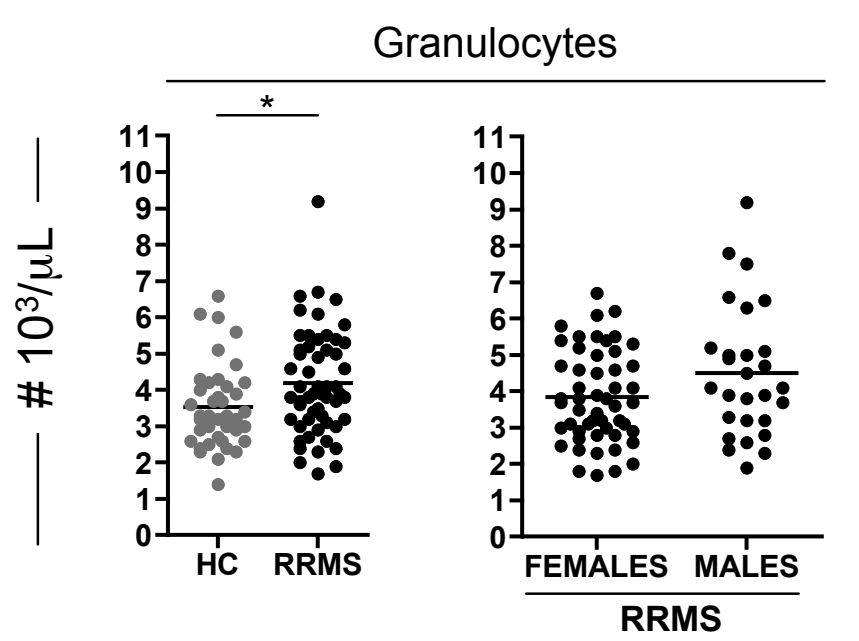

CXCR1

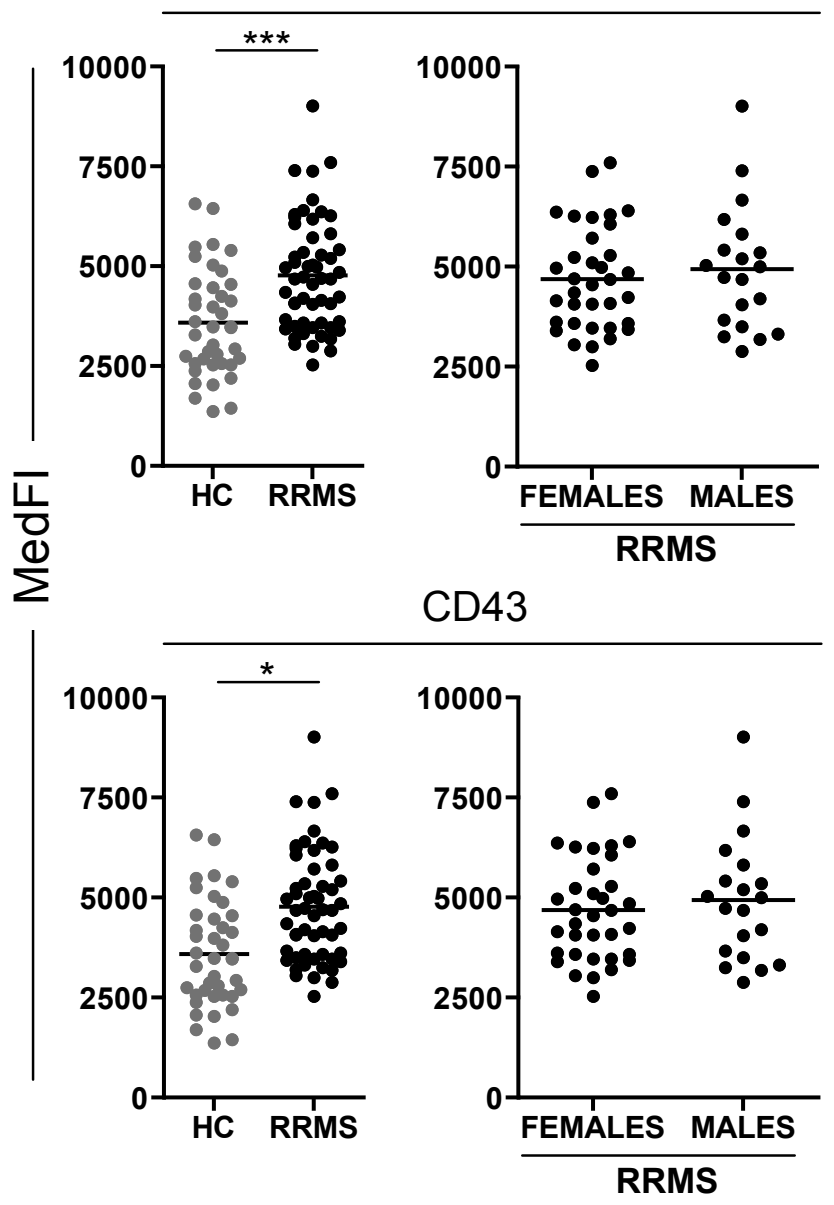

FPR1

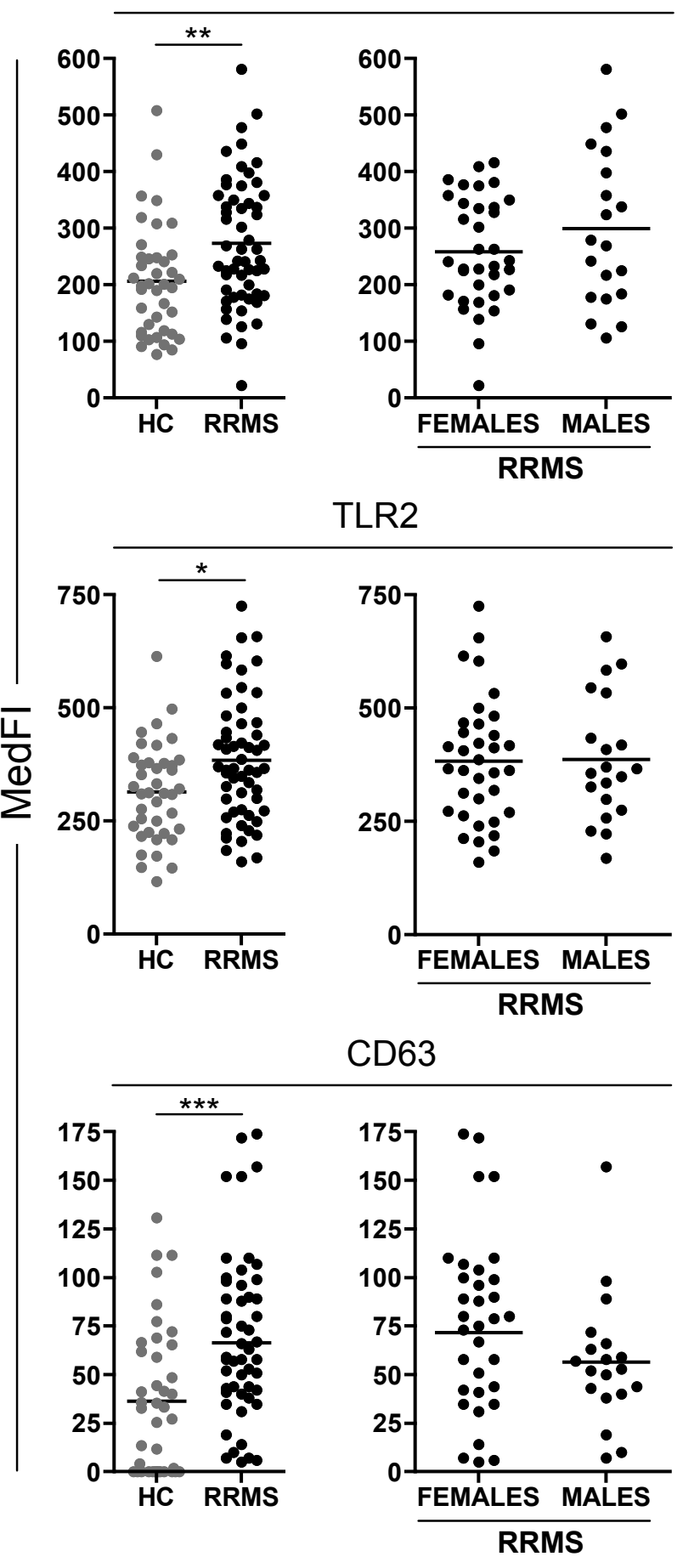

